\title{
Multi-generation massive star-formation in NGC 3576
}

\author{
C. R. Purcell ${ }^{1,2}$, V. Minier ${ }^{3,4}$, S. N. Longmore ${ }^{2,5,6}$, Ph. André ${ }^{3,4}$, A. J. Walsh ${ }^{2,7}$, P. Jones ${ }^{2,8}$, F. Herpin ${ }^{9,10}$, T. Hill ${ }^{2,11,12}$, \\ M. R. Cunningham ${ }^{2}$, and M. G. Burton ${ }^{2}$
} \author{
1 Jodrell Bank Centre for Astrophysics, Alan Tur
Oxford Road, Manchester M13 9PL, UK
e-mail: cormac.purcell@manchester.ac.uk \\ 2 School of Physics, University of New South Wales, Sydney, NSW 2052, Australia \\ 3 CEA, DSM, IRFU, Service d'Astrophysique, 91191 Gif-sur-Yvette, France \\ 4 Laboratoire AIM, CEA/DSM - CNRS - Université Paris Diderot, IRFU/Service d'Astrophysique, CEA-Saclay, \\ 91191 Gif-sur-Yvette, France \\ 5 Harvard-Smithsonian Centre For Astrophysics, 60 Garden Street, Cambridge, MA, 02138, USA \\ ${ }^{6}$ CSIRO Australia Telescope National Facillity, PO Box 76, Epping, NSW 1710, Australia \\ 7 Centre for Astronomy, James Cook University, Townsville, QLD 4811, Australia \\ 8 Departmento de Astronoma, Universidad de Chile, Casilla 36-D, Santiago, Chile \\ 9 Université de Bordeaux, Laboratoire d'Astrophysique de Bordeaux, 33000 Bordeaux, France \\ 10 CNRS/INSU, UMR 5804, BP 89, 33271 Floirac Cedex, France \\ 11 School of Physics, University of Exeter, Stocker Road, EX4 4QL, Exeter, UK \\ 12 Leiden Observatory, Leiden University, PO BOX 9513, 2300 RA Leiden, the Netherlands
}

Received 16 November 2008 / Accepted 3 July 2009

ABSTRACT

\begin{abstract}
Context. Recent 1.2-mm continuum observations have shown the giant $\mathrm{H}$ II region NGC 3576 to be embedded in the centre of an extended filamentary dust-cloud. The bulk of the filament away from the H II region contains a number of clumps seen only at (sub-)millimetre wavelengths. Infrared and radio observations of the central star cluster have uncovered evidence of sequential starformation leading us to believe that the adjacent clumps may host massive protostellar objects at a very early stage of evolution.

Aims. We have investigated the physical and chemical conditions in the dusty clumps with the goal of characterising their star-forming content.

Methods. We have used the Australia Telescope Compact Array (ATCA) to image the cloud for the $\mathrm{NH}_{3}(1,1),(2,2)$ and $(4,4)$ transitions, $22 \mathrm{GHz} \mathrm{H}_{2} \mathrm{O}$ masers, and $23 \mathrm{GHz}$ continuum emission. The 70-m Tidbinbilla dish was used to estimate the total integrated intensity of $\mathrm{NH}_{3}$. We also utilised the 22-m Mopra antenna to map the region for the molecular lines ${ }^{13} \mathrm{CO}(1-0), \mathrm{C}^{18} \mathrm{O}(1-0)$, $\mathrm{HCO}^{+}(1-0), \mathrm{H}^{13} \mathrm{CO}^{+}(1-0), \mathrm{CS}(1-0)$ and $\mathrm{N}_{2} \mathrm{H}^{+}(1-0)$.

Results. Emission from dense molecular gas follows the morphology of the 1.2-mm dust emission, except towards the central ionised region. The $\mathrm{H}$ II region is observed to be expanding into the molecular cloud, sweeping up a clumpy shell of gas, while the central star cluster is dispersing the molecular gas to the east. Analysis of the $\mathrm{NH}_{3}$ data indicates that temperature and linewidth gradients exist in the western arm of the filament. Temperatures are highest adjacent to the central $\mathrm{H}$ II region, indicating that the embedded cluster of young stars there is heating the gas. Six new $\mathrm{H}_{2} \mathrm{O}$ masers were detected in the arms of the filament, all associated with $\mathrm{NH}_{3}$ emission peaks, confirming that star-formation has begun within these cores. Core masses range from 5 to $516 M_{\odot}$ and most appear to be gravitationally bound. Complementary results by André et al. (2008) imply that seven cores will go on to form massive stars between 15 and $50 M_{\odot}$. The large scale velocity structure of the filament is smooth, but at least one clump shows the signature of inward gas motions via asymmetries in the $\mathrm{NH}_{3}(1,1)$ line profiles. The same clump exhibits an enhanced abundance of $\mathrm{N}_{2} \mathrm{H}^{+}$, which coupled with an absence of $\mathrm{CO}$ indicates depletion onto the dust grain surface.

Conclusions. The HII region at the heart of NGC 3576 is potentially triggering the formation of massive stars in the bulk of the associated cloud.
\end{abstract}

Key words. ISM: molecules - stars: formation - HII regions - radio lines: ISM - ISM: abundances - stars: pre-main-sequence

\section{Introduction}

\subsection{Triggered star-formation}

A necessary precursor to massive star formation is the existence of dense clumps of self-gravitating gas. Elmegreen (1998) developed the first coherent picture of sequential star formation, in which the formation and collapse of these clumps within giant molecular clouds is triggered by an external event. Three distinct triggering mechanism are considered:

1. globule squeezing: compression of pre-existing clumps, e.g. due to a propagating shock-wave from a supernova;
2. cloud-cloud collisions: two molecular clouds collide resulting in gravitational instabilities;

3. collect and collapse: accumulation of gas into a shell or ridge, and subsequent fragmentation and collapse.

Examples of the third mechanism are observed to occur on the edges of $\mathrm{H}$ II regions. Created by the far-UV radiation from a young OB-cluster, the hot ionised gas expands rapidly, sweeping up a shell of dense molecular material before it. A photon dominated region (PDR) is created at the interface between the $\mathrm{H}$ II region and the molecular cloud (see Hollenbach \& Tielens 1999 for a review of PDRs). If the PDR is over-pressured 


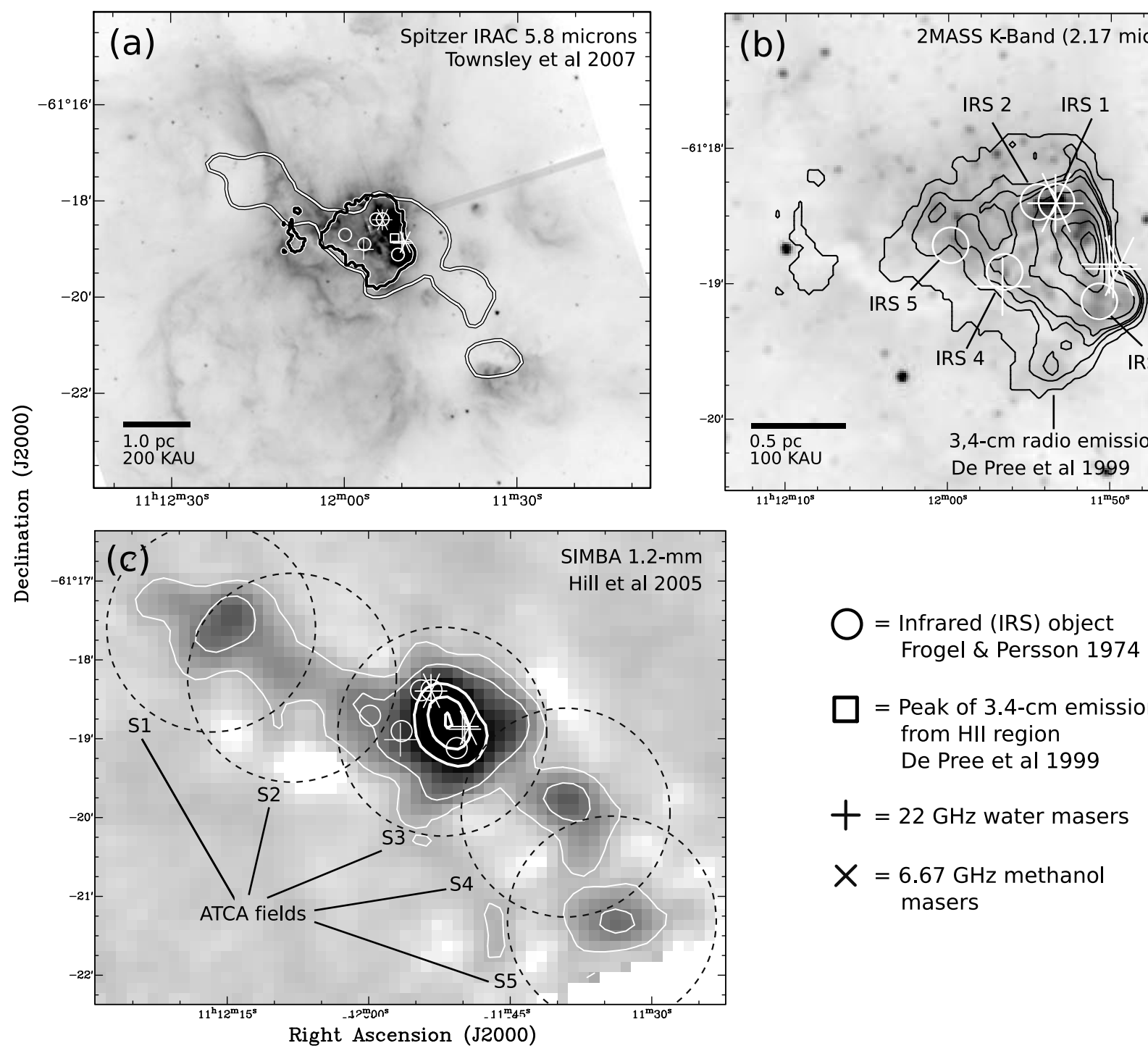

Fig. 1. Overview of the NGC 3576 star forming region. a) SPITZER $5.8 \mu \mathrm{m}$ image showing the extent of the 3.4-cm radio emission (black contour, $\sim 3$ percent) and the 1.2-mm continuum emission (white contour, $\sim 5$ percent). $\mathrm{CH}_{3} \mathrm{OH}$ and $\mathrm{H}_{2} \mathrm{O}$ maser positions are marked with " $\times$ " and "+" symbols, respectively. Circles mark the positions assigned to the original infrared sources (IRS) discovered by Frogel \& Persson (1974), while a " $\square$ " marks the peak of the radio-continuum emission; b contours of the 3.4-cm free-free emission from ionised gas in the giant $\mathrm{H}$ II region at the heart of the complex (de Pree et al. 1999) plotted over the $2.2 \mu \mathrm{m} K$-band image from 2MASS; c) 1.2-mm continuum image of the complex (Hill et al. 2005) showing the dusty filament running across the $\mathrm{H}$ II region. Dashed circles indicate the five fields observed with the ATCA, centred on the $1.2-\mathrm{mm}$ clumps.

compared to the bulk of the molecular gas, shocks are driven into the dense neutral medium of the cloud, potentially leading to its fragmentation into clumps (e.g. Urquhart et al. 2006 and references therein). Subsequent disturbances may then trigger collapse and hence star formation. Material swept into a clump shields the column of molecular material behind it, forming giant pillars, the best known example of which are the "elephant trunks" in the Eagle Nebula (White et al. 1999; Allen et al. 1999). Some observed examples of swept-up shells around $\mathrm{H}$ II regions include Sh2-212 (Deharveng et al. 2008), RCW120 (Deharveng et al. 2009), W5 (Karr \& Martin 2003), RCW 49 (Whitney et al. 2004), and RCW 79 (Zavagno et al. 2006).

It is important to note that energy injected into molecular clouds by newly formed stars may instead quench the star formation process. Supersonic particle-winds from massive stars account for $\sim 0.1-1.0$ per cent of the stellar luminosity and act to disperse molecular material and dust (e.g., Genzel 1991). The two most important feedback mechanisms, photo-ionisation and cluster winds, compete with each other to disrupt the cloud. On the scale of giant molecular clouds, feedback from newly formed stars is responsible for regulating the star-formation rate and hence the evolution of Galactic structure.

In this paper we present new multi-wavelength observations of the giant $\mathrm{H}$ II region NGC 3576, which is thought to be undergoing sequential star formation (see Sect. 1.2). We examine the evidence for star formation in the nearby molecular environment.

\subsection{Prior observations of NGC 3576}

The environment of the giant $\mathrm{H}$ II region NGC 3576 (also known as RCW 57, G291.3-0.7 and IRAS 11097-6102) has been extensively studied at infrared and radio wavelengths. Figure 1a presents the $5.8 \mu \mathrm{m}$ SPITZER IRAC image of the whole 
star-forming complex with the $\mathrm{H}$ in region at the centre. The hourglass shaped emission feature running north-south across the centre of the image likely correspond to a bipolar cavity being evacuated by the central embedded cluster. The giant molecular cloud which hosts NGC3576 manifests itself as large extincted areas to the north-east and south-west, and is at a distance of $2.4 \mathrm{kpc}$ (Persi et al. 1994). The extent of the $\mathrm{H}$ II region is illustrated by the thick black contour, corresponding to the 3- $\sigma$ (54 mJy/beam) level in the 3.4-cm free-free emission mapped by de Pree et al. (1999). Figure $1 \mathrm{~b}$ shows the distribution of the 3.4-cm emission plotted over the $2.17 \mu \mathrm{m}$ infrared image from 2MASS. The ionised gas covers the brightest infrared emission, peaking sharply in the west and extending $\sim 2^{\prime}$ to the north-east and south. Frogel \& Persson (1974) discovered five near- and mid-infrared sources towards the ionised gas (plotted as circles in Fig. 1), with the brightest source (IRS 1) located adjacent to the main peak of the radio emission. Further high resolution photometric observations by Persi et al. (1994) have revealed the presence of a very young, deeply embedded cluster with 130 members in the same region. Spectra of these sources exhibit deep $9.7 \mu \mathrm{m}$ silicate absorption features (Moorwood \& Salinari 1981), leading to their interpretation as pre-main-sequence objects.

There is controversial evidence for sequential star formation in NGC 3576. Persi et al. (1994) showed that a steep near-IR colour gradient exists in the embedded cluster, with the reddest, most deeply embedded sources in the west. This implies that star-formation began in the east, gradually moving to the location of the strong radio peak in the west. Such an interpretation is supported by the existence of an electron temperature gradient in the ionised gas (de Pree et al. 1999) indicating that the youngest and hottest stars are located near the sharp western edge of the $\mathrm{H}$ II region (Hjellming 1966). High helium abundance in the east may also indicate the presence of a population of older stars and their mass loss (Hanson \& Conti 1993). Bright $22 \mathrm{GHz}$ water masers, commonly found in outflows, have been found adjacent to the main radio peak (Caswell 2004), while Norris et al. (1993) also detected two $6.67 \mathrm{GHz}$ methanol maser sites near IRS 1 and IRS 3. Shocked molecular hydrogen emission was measured peaking towards IRS 1 by Oliva \& Moorwood (1986), also pointing to the existence of outflows. IRS 1 was later resolved into three components by Moneti (1992) and Barbosa et al. (2003), one of which is seen through scattered light from a cavity or a dusty disk (Moneti 1992). None of the detected infrared sources contributes significantly to the ionisation of the $\mathrm{H}$ il region, (Barbosa et al. 2003), however, recent Chandra observations (Townsley 2006) have revealed multiple deeply embedded hard X-ray sources, which may provide the extra ionisation needed.

The $\mathrm{H}$ II region was mapped at 1-mm wavelengths for continuum emission, with a resolution of $1^{\prime}$, by Cheung et al. (1980), who found that the flux density ratio compared to 40-350 $\mu \mathrm{m}$ was consistent with optically thin thermal dust emission. More recently, Hill et al. (2005) used the SIMBA ${ }^{1}$ bolometer to map 1.2- $\mathrm{mm}$ continuum emission at a resolution of $\sim 24^{\prime \prime}$, as shown in Fig. 1c and via white contours in Fig. 1a. The SIMBA field covers an area of $4^{\prime} \times 6^{\prime}$, centred on the radio peak and reveals that the $\mathrm{H}_{\mathrm{II}}$ region is embedded in a filamentary structure of cool dust, running north-east to south-west. The H II region is prominent at the centre, however, several bright knots are apparent along the length of the filament. These clumps,

${ }^{1}$ Sest IMaging Bolometer Array on the Sweedish ESO Submillimeter Telescope. designated S1-S5 in this work, fall along the dark lane traversing the nebulosity in the near-infrared image, and are coincident with infrared-dark clouds (IRDCs) in the mid-infrared (e.g., the $5.8 \mu \mathrm{m}$ IRAC image). IRDCs have been found throughout the Galactic plane with masses in excess of $30 M_{\odot}$ and it has been suggested they are the cold precursors to massive star clusters (e.g., Pillai et al. 2006; Rathborne et al. 2006).

NGC 3576 constitutes an ideal laboratory in which to study the process of massive star formation. In particular, we aim to investigate if the $\mathrm{H}$ II region is interacting with the dusty filament and if star-formation has been triggered in the dense knots along its length. Here we present new observations of the whole filament in several molecular tracers, with the goal of constraining the star-forming properties of these new clumps (S1, S2, S4 \& $\mathrm{S} 5$ ), and determining the effect of the $\mathrm{H}$ in region on the complex.

\section{Observations and data reduction}

Data from three telescopes, the Australia Telescope Compact Array (ATCA), the 22-m Mopra telescope and the 70-m Tidbinbilla telescope, were combined to assemble a picture of NGC 3576. The ATCA was used to obtain high resolution $\mathrm{NH}_{3}$ maps as a probe of the density and temperature structure of the clumps within the filament. We simultaneously searched for $\mathrm{UCH}$ II regions via their $23 \mathrm{GHz}$ free-free continuum emission. We used the Tidbinbilla telescope to map the extended $\mathrm{NH}_{3}$ emission, providing a measure of the total column of $\mathrm{NH}_{3}$ in the filament and an estimation of the "missing flux" in the interferometer images. We utilised the Mopra telescope to map the complex in the $3-\mathrm{mm}$ lines ${ }^{13} \mathrm{CO}(1-0), \mathrm{C}^{18} \mathrm{O}(1-0)$, $\mathrm{HCO}^{+}(1-0), \mathrm{H}^{13} \mathrm{CO}^{+}(1-0), \mathrm{N}_{2} \mathrm{H}^{+}(1-0)$ and $\mathrm{CS}(2-1)$, with the goal of probing the physical and chemical conditions in each of the dusty clumps. Line rest frequencies and electronic constants for each transition are noted in Table A.1 in the Appendix.

\subsection{ATCA observations}

Observations were made with the ATCA in three blocks during the years 2003-2005. Table 1 summarises details of the dates, array-configurations and frequencies used. $\mathrm{NH}_{3}(1,1)$ data was obtained using the EW367 and H75 array configurations in August 2003 and July 2005, respectively. Two orthogonal linear polarisations were observed at each frequency. The raw data were processed in a correlator, which was configured to deliver a bandwidth of $\sim 101 \mathrm{~km} \mathrm{~s}^{-1}$ split into $\sim 0.2 \mathrm{~km} \mathrm{~s}^{-1}$ wide channels. $\mathrm{NH}_{3}(2,2)$ was observed simultaneously with $\mathrm{NH}_{3}(1,1)$ on the H75 array in July 2005. Due to technical constraints only 128 channels were available on the correlator, yielding a spectral resolution of $\sim 0.8 \mathrm{~km} \mathrm{~s}^{-1}$ over the $101 \mathrm{~km} \mathrm{~s}^{-1}$ bandwidth. In August 2003 the region was also mapped for $23 \mathrm{GHz}$ continuum and $22 \mathrm{GHz}$ water masers using the EW367 array. In July 2004, the $750 \mathrm{D}$ array was used to simultaneously map the $\mathrm{NH}_{3}(4,4)$ transition and $23 \mathrm{GHz}$ continuum. The correlator configuration was identical to that used to obtain the $\mathrm{NH}_{3}(1,1)$ data.

The primary beam of the ATCA at $23 \mathrm{GHz}$ has a full width half maximum $(F W H M)$ of $2.5^{\prime}$. In order to cover the extent of the $1.2 \mathrm{~mm}$ continuum emission we observed five overlapping fields, marked by dashed circles in Fig. 1d and centred approximately on the coordinates of the dusty SIMBA clumps. Table 2 lists the coordinates of the pointing centres. Each of the five fields was observed for $10 \mathrm{~min}$ in turn, over the course of one hour and this pattern was repeated for $10 \mathrm{~h}$, giving a total of $1.7 \mathrm{~h}$ on each position. In order to correct for fluctuations in the phase and amplitude caused by atmospheric and instrumental effects, a 
Table 1. Details of the Australia Telescope Compact Array observing runs.

\begin{tabular}{|c|c|c|c|c|c|c|c|}
\hline $\begin{array}{l}\text { UT } \\
\text { date }\end{array}$ & $\begin{array}{l}\text { Array } \\
\text { config. }\end{array}$ & $\begin{array}{l}\text { Beam }^{\alpha} \\
\text { size (") }\end{array}$ & Transitions & $\begin{array}{c}\text { Frequency } \\
(\mathrm{GHz})\end{array}$ & $\begin{array}{c}\text { Bandwidth } \\
\text { (MHz) }\end{array}$ & $\begin{array}{c}\text { No. } \\
\text { channels }\end{array}$ & $\mathrm{K} /(\mathrm{Jy} / \mathrm{bm})$ \\
\hline \multirow[t]{3}{*}{2003 Aug. 28} & EW367 & $7.6 \times 6.2$ & $\mathrm{NH}_{3}(1,1)$ & 23.694480 & 8 & 512 & $46.02^{\beta}$ \\
\hline & & & Continuum & 23.694500 & 128 & 32 & - \\
\hline & & & $\mathrm{H}_{2} \mathrm{O}(6-5)$ & 22.235120 & 16 & 16 & - \\
\hline \multirow[t]{2}{*}{2004 Jul. 02} & $750 \mathrm{D}$ & $6.6 \times 2.4$ & $\mathrm{NH}_{3}(4,4)$ & 24.139417 & 8 & 512 & 134.47 \\
\hline & & & Continuum & 22.000000 & 128 & 32 & - \\
\hline \multirow[t]{2}{*}{2005 Jul. 17} & $\mathrm{H} 75$ & $26.8 \times 20.9$ & $\mathrm{NH}_{3}(1,1)$ & 23.694480 & 8 & 512 & 3.89 \\
\hline & & & $\mathrm{NH}_{3}(2,2)$ & 23.722630 & 8 & 128 & 3.88 \\
\hline
\end{tabular}

${ }^{\alpha}$ Beam size excluding the $3 \mathrm{~km}+$ baselines to Antenna $6 ;^{\beta}$ Jy to $\mathrm{K}$ conversion factor for the combined EW367 and $\mathrm{H} 75 \mathrm{data}$ is $17.58 \mathrm{~K} /(\mathrm{Jy} / \mathrm{bm})$.

Table 2. Centre coordinates of the ATCA fields corresponding approximately to the peak $1.2-\mathrm{mm}$ emission.

\begin{tabular}{lcc}
\hline \hline Field & $\begin{array}{c}\text { RA } \\
(\mathrm{J} 2000)\end{array}$ & $\begin{array}{c}\text { Dec } \\
(\mathrm{J} 2000)\end{array}$ \\
\hline S1 & $11^{\mathrm{h}} 12^{\mathrm{m}} 17.00^{\mathrm{s}}$ & $-61^{\mathrm{d}} 17^{\mathrm{m}} 40.0^{\mathrm{s}}$ \\
S2 & $11^{\mathrm{h}} 12^{\mathrm{m}} 04.90^{\mathrm{s}}$ & $-61^{\mathrm{d}} 18^{\mathrm{m}} 20.0^{\mathrm{s}}$ \\
S3 & $11^{\mathrm{h}} 11^{\mathrm{m}} 51.50^{\mathrm{s}}$ & $-61^{\mathrm{d}} 18^{\mathrm{m}} 52.0^{\mathrm{s}}$ \\
S4 & $11^{\mathrm{h}} 11^{\mathrm{m}} 38.33^{\mathrm{s}}$ & $-61^{\mathrm{d}} 19^{\mathrm{m}} 53.9^{\mathrm{s}}$ \\
S5 & $11^{\mathrm{h}} 11^{\mathrm{m}} 33.86^{\mathrm{s}}$ & $-61^{\mathrm{d}} 21^{\mathrm{m}} 20.9^{\mathrm{s}}$ \\
\hline
\end{tabular}

strong phase calibrator was observed for two minutes before and after changing fields. The instrumental contribution to the bandpass shape was measured by integrating on a strong continuum source (e.g., 0420-014) and was subsequently subtracted from all spectra. A primary flux calibrator (1934-638 or Uranus) was observed once per observation period, to allow the absolute calibration of the flux scale.

The data were reduced using the MIRIAD package (Sault et al. 1995) following standard procedures. During the data reduction the sources were assumed to be unpolarised and both polarisations were averaged together. Continuum emission was subtracted from the spectral line data by using the task uvlin to fit a polynomial to the line free-channels. An image of the continuum emission was produced by combining the line-free channels in the $\mathrm{NH}_{3}$ data with the dedicated wide-band 22 and $23 \mathrm{GHz}$ continuum observations. Images were made using the invert task and natural weighting was used to minimise the noise in the imageplane. At this stage the $\sim 3 \mathrm{~km}$ baselines to antenna CA06 were discarded as the phases were found to be decorrelated, degrading the image quality. All images were deconvolved using the standard clean algorithm and, if a sufficiently bright source was present, several iterations of the selfcal task were applied. The above procedure was performed on all fields, before using the task linmos to mosaic the data into a single map. Finally the $\mathrm{NH}_{3}$ data were converted to a brightness temperature scale in Kelvin, using the Jy/K scaling factors noted in Table 1.

\subsection{Mopra observations}

The Mopra observations were conducted in "on the fly" (OTF) mapping mode, between the months June-September, during 2004 and 2005. An image was built up by combining overlapping scan rows, each containing 30-40 spectra. Scan rows were offset by half of the beam- $F W H M$ and the scanning speed was slow enough to ensure Nyquist sampling in the scan direction.

NGC 3576 was divided into an overlapping mosaic of three or more $5^{\prime} \times 5^{\prime}$ fields, positioned to cover the $1.2-\mathrm{mm}$ continuum emission. The pointing centres of individual fields varied, depending on where molecular emission was detected, and the final maps were assembled by co-adding individual fields together. Each field took $\sim 80$ min to complete, plus a further $\sim 10$ min for pointing checks and calibration measurements.

The signal from the receiver was processed in a digital autocorrelator, configured to have a bandwidth of $64 \mathrm{MHz}$ divided into 1024 channels, which provided a velocity resolution of $\sim 0.2 \mathrm{~km} \mathrm{~s}^{-1}$ over a usable bandwidth of $\sim 120 \mathrm{~km} \mathrm{~s}^{-1}$. The central frequency was chosen so that channel 512 was centred on the systemic velocity of NGC 3576 at $-24 \mathrm{~km} \mathrm{~s}^{-1}$. Observations were made in dual orthogonal linear polarisation mode and the polarisations were averaged together during the reduction procedure. The pointing accuracy was checked using a nearby $\mathrm{SiO}$ maser before observing each field and was estimated to be better than $8^{\prime \prime}$. Calibration to the $T_{\mathrm{A}}^{*}$ scale was achieved by measuring the emission from a single hot load placed in front of the receiver every 20 min (see Ladd et al. 2005 and Kutner \& Ulich 1981). The maps were further calibrated onto the main-beam brightness temperature scale $\left(T_{\mathrm{MB}}\right)$ by dividing the $T_{\mathrm{A}}^{*}$ pixel values by the main beam efficiency $\eta_{\mathrm{mb}}$ at the observing frequency (see Table 4 of Ladd et al. 2005).

The data were reduced using the LIVEDATA and GRIDZILLA packages, available from the ATNF ${ }^{2}$. LIVEDATA performed bandpass calibration by subtracting the preceding OFF spectrum from the SCAN spectra in each row. A low-order polynomial was then fit to line-free channels and subtracted, resulting in a smooth baseline at zero Kelvin. The spectra were assigned individual position stamps and regridded to the LSR-K reference frame, before being written to disk. The GRIDZILLA package was then used to resample the maps to a regular pixel scale, weighted according to the system temperature $\left(T_{\text {sys }}\right)$. To grid the data we used a pixel-size of $6 \times 6$ arcseconds and a Gaussian smoothing kernel with a $F W H M$ of $18^{\prime \prime}$, truncated at an angular offset of $36^{\prime \prime}$. The final data cubes were smoothed to an angular resolution of $\sim 40^{\prime \prime}$.

\subsection{Tidbinbilla observations}

The 70-m antenna located at the NASA Tidbinbilla Deep Space Communications Complex near Canberra has a limited time devoted to radio astronomy. When Mars is above the horizon the 70-m telescope is almost entirely utilised supporting the various NASA missions to that planet and as such has been little used by the radio astronomy community. In 2005 we performed the first frequency-switched spectral line mapping observations using the $23 \mathrm{GHz} K$-band receiver. Fast frequency switching also provides better cancellation of atmospheric emission fluctuations, however, the response of the receiver may vary considerably with frequency, resulting in poor spectroscopic baselines.

\footnotetext{
${ }^{2}$ http://www.atnf.csiro.au/computing/software/
} 

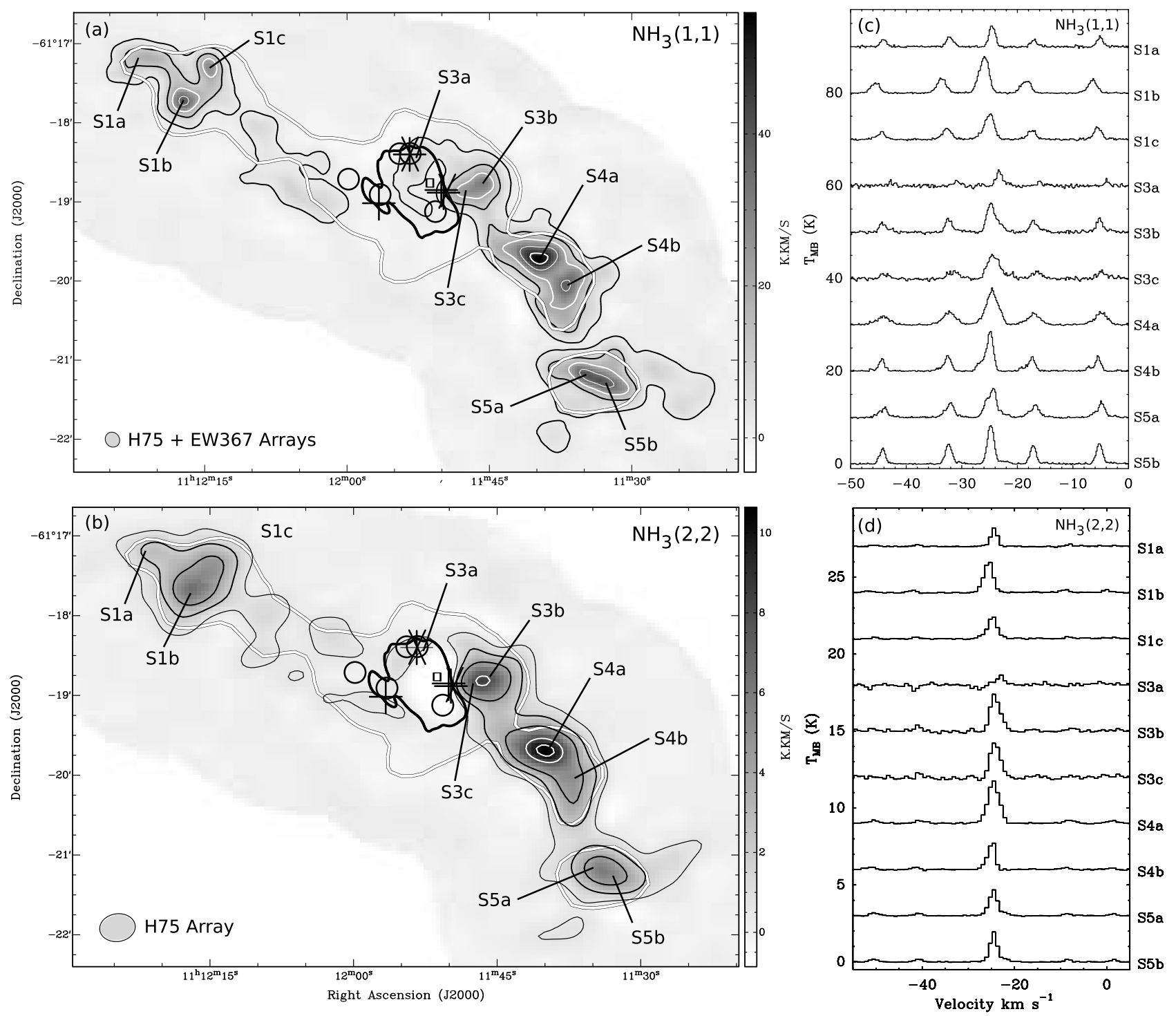

Fig. 2. a) Integrated intensity map of the $\mathrm{NH}_{3}(1,1)$ emission (thin black and white contours, and greyscale) made using the combined ATCA H75 and EW367 data. Contours are at 1.6, 7.9, 13.8, 20.0, 25.7, 31.5, 37.4, 43.6 and 50.1 K. The extent of the $23 \mathrm{GHz}$ continuum emission from the central $\mathrm{H}$ II region is marked by the thick black contour. The 1.2-mm dust emission is outlined by a single white contour $(\sim 3 \mathrm{Jy} /$ beam, from Hill et al. 2005) and matches the $\mathrm{NH}_{3}$ emission closely, except in the central clump. Crosses (+) mark the positions of $22 \mathrm{GHz}$ water masers while " $\times$ " symbols mark the $6.67 \mathrm{GHz}$ methanol maser sites. IRS sources are marked by circles; b) integrated intensity map of the $\mathrm{NH}_{3}(2,2)$ emission made using data from the ATCA $\mathrm{H} 75$ array; c) sample $\mathrm{NH}_{3}(1,1)$ spectra measured from the positions labelled S1a-S5b. Spectra have been offset from zero baseline for clarity. The brightness temperature ratios between the main and satellite lines indicate that the clumps have low to moderate optical depths; d) sample $\mathrm{NH}_{3}(2,2)$ spectra measured from the same positions.

We targeted the same five fields in NGC 3576 as were observed with the ATCA. The OTF method had not yet been implemented on the 70-m antenna so we observed each field as a square grid of $5 \times 5$ positions, spaced by half the $45^{\prime \prime}$ beamFWHM. Each position was observed for $10 \mathrm{~min}$ and the pointing accuracy was checked by observing an unresolved planet (Jupiter) every hour. Pointing errors were usually below $10^{\prime \prime}$ or $1 / 4$ beam. Individual maps took 5-6 h to complete, including 15 min to measure the atmospheric opacity using the "skydip" method. During the observations the data were calibrated against an ambient load (a noise diode) and the zenith system temperature at $23 \mathrm{GHz}$ was typically $40-50 \mathrm{~K}$ during the observations. We estimate the $T_{\mathrm{A}}^{*}$ flux scale is uncertain by $\leq 10$ per cent. The peak aperture efficiency was measured as $0.48 \pm 5 \%$ at $22.2 \mathrm{GHz}$ by Greenhill et al. (2003) and we adopt that value here to correct the data onto the main beam brightness temperature flux scale $\left(T_{\mathrm{MB}}\right)$. To convert to units of Jy/beam the data were multiplied by $0.951(\mathrm{Jy} / \mathrm{beam}) / \mathrm{K}$.

The $K$-band receiver on the 70-m antenna measured only the left circular polarisation. The bandpass was centred mid-way between the $\mathrm{NH}_{3}(1,1)$ and $(2,2)$ transitions and the correlator was configured to have a $64 \mathrm{MHz}$ wide bandpass divided into 2048 channels. Both the $\mathrm{NH}_{3}(1,1)$ and $(2,2)$ spectra, including satellite lines, fell well inside the $850 \mathrm{~km} \mathrm{~s}^{-1}$ usable range and were observed at a velocity resolution of $\sim 0.4 \mathrm{~km} \mathrm{~s}^{-1}$.

The data were reduced in an ad-hoc pipeline built using the SPC, GILDAS and MIRIAD packages. SPC was used initially to quotient and resample the spectra to a common $V_{\mathrm{LSR}}$ rest-frame. 
The spectra were inverted and frequency-shifted in the CLASS package. As the spectral baselines were poor, a polynomial of order 5-9 (in extreme cases) was fit to the line-free channels before spectra from individual integrations were averaged together. The data were then assembled into a $3 \mathrm{D}$ data-cube using the cube command in the GREG package and resampled onto a finer spatial pixel grid using the fill_cube command. Finally, the data were output as fits cubes and MIRIAD was used to restore missing header information, such as the beam-size and data-unit.

\section{Results}

Molecular emission was detected from all of the clumps identified in the SIMBA map. The following subsections describe the results from the ATCA, Tidbinbilla and Mopra telescopes separately.

\subsection{ATCA results}

We detected $\mathrm{NH}_{3}(1,1)$, and $\mathrm{NH}_{3}(2,2)$ across the whole filament. $\mathrm{NH}_{3}(4,4)$ was not detected down to a sensitivity limit of $\sim 8 \mathrm{mJy} /$ beam $(1.0 \mathrm{~K})$ when the data were smoothed to a velocity resolution of $1 \mathrm{~km} \mathrm{~s}^{-1}$. The $J, K=(4,4)$ level is excited by gas above $200 \mathrm{~K}$ (see Fig. 1 in Ho \& Townes 1983), hence no substantial reservoirs of hot gas are present in NGC 3576. Figures $2 \mathrm{a}$ and $\mathrm{b}$ present the $\mathrm{NH}_{3}(1,1)$ and $(2,2)$ integrated intensity maps of the region. The $\mathrm{NH}_{3}(1,1)$ map utilises data from both the $\mathrm{H} 75$ and EW367 array configurations and has a resolution of $\sim 11^{\prime \prime}$. Only $\mathrm{H} 75$ data exist for the $\mathrm{NH}_{3}(2,2)$ transition and the resulting map has a resolution of $\sim 23^{\prime \prime}$. For reference, the 5 per cent contour $(0.3 \mathrm{Jy} /$ beam $)$ of the SIMBA $1.2-\mathrm{mm}$ continuum emission is plotted in white. The thick black line is the 3 per cent contour $(0.11 \mathrm{Jy} / \mathrm{beam})$ of the $23-\mathrm{GHz}$ continuum emission from the central $\mathrm{H}$ II region. We see the $\mathrm{NH}_{3}$ emission has approximately the same morphology in both transitions and closely follows the structure of the 1.2-mm dust emission, except immediately to the east of the central $\mathrm{H}_{\text {II }}$ region (SIMBA clumps S2 \& S3). The 1.2$\mathrm{mm}$ emission in these clumps is over 50 percent contaminated by free-free emission (see André et al. 2008). Sample $\mathrm{NH}_{3}(1,1)$ and $(2,2)$ spectra from peak positions on the integrated intensity maps (labelled S1a-S5b) are presented in Figs. 2c and -d. Both of the $\mathrm{NH}_{3}$ transitions exhibit the classic "five-finger" profiles at all positions. Some asymmetries between the $\mathrm{NH}_{3}(1,1)$ satellite lines are evident towards all positions, however, for the most part the spectra appear optically thin, exhibiting optical depths between $\tau=0.1$ and $\tau=2.0$. Linewidths vary between 1 and $2.5 \mathrm{~km} \mathrm{~s}^{-1}$, and peak brightness temperatures range between 4.4 and $8 \mathrm{~K}$ for the $\mathrm{NH}_{3}(1,1)$ line and between 1.2 and $2.6 \mathrm{~K}$ for the $\mathrm{NH}_{3}(2,2)$ lines. Individual line profiles in the western arm of the filament show clear evidence of blending and multiple components, especially towards positions S3b, S3c, S4b and S5a.

$23 \mathrm{GHz}$ continuum was observed simultaneously with each of the $\mathrm{NH}_{3}$ lines. To make the final map we combined data from the EW367, H75 and 750D arrays, including the continuum visibilities from the line-free channels in the narrow-band spectral line windows. No $23 \mathrm{GHz}$ continuum emission was detected outside of the central HII region, down to a sensitivity limit of $\sim 0.5 \mathrm{mJy} /$ beam. Figure 3 presents the image of the $\mathrm{H}_{\text {II }}$ region at $23 \mathrm{GHz}$. " $\times$ " and "+" symbols mark the positions of known $6.67 \mathrm{GHz}$ methanol masers and $22 \mathrm{GHz}$ water masers, respectively (Norris et al. 1993; Caswell 2004). The contours are similar to the central part of the 3.4-cm map made by de Pree et al. (1999) (see Fig. 1b), with a single strong peak at $11^{\mathrm{h}} 11^{\mathrm{m}} 51.08^{\mathrm{s}}$, $-61^{\mathrm{d}} 18^{\mathrm{m}} 50.0^{\mathrm{s}}(\mathrm{J} 2000)$ and diffuse emission extending eastward

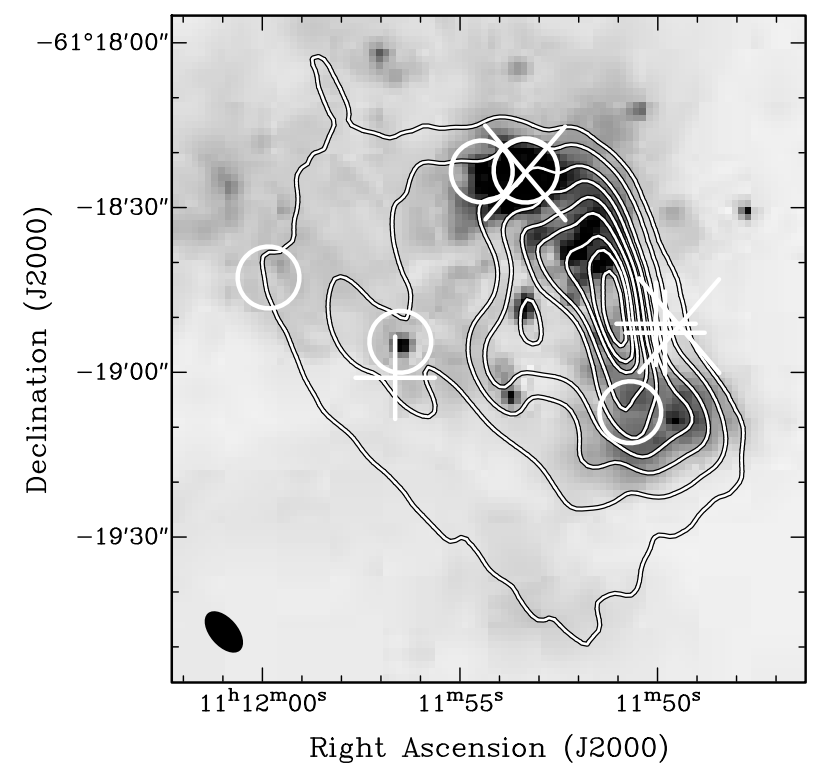

Fig. 3. 23-GHz continuum emission from the $\mathrm{H}$ II region at the centre of the NGC 3576 complex (contours) overlaid on the $3.6 \mu$ m SPITZER IRAC image (greyscale). The continuum image was made by combining multi-frequency data from the ATCA H75, 750D and EW367 arrays, including the line-free channels from the $\mathrm{NH}_{3}$ observations. Contours are at values of $0.01,0.110 .45,0.85,1.35,1.65,2.00$, $2.48,2.82$, and $3.25 \mathrm{Jy} /$ beam. "+" and " $\times$ " symbols mark $\mathrm{H}_{2} \mathrm{O}$ and $\mathrm{CH}_{3} \mathrm{OH}$ masers, respectively, while circles mark the positions of the Frogel \& Persson (1974) IRS sources. The total integrated flux density is $\sim 42.6 \mathrm{Jy}$.

into SIMBA clump S2. The $23 \mathrm{GHz}$ flux density at the peak is $3.6 \mathrm{Jy} /$ beam and we measure a total integrated intensity of approximately $42.6 \mathrm{Jy}$. By comparison, the total flux at $3.4-\mathrm{cm}$ is measured to be $71 \mathrm{Jy}$. Our observations sample the $u v$-plane less well than de Pree et al. (1999) and we likely filter out a significant fraction of the extended emission. Our values are consistent with free-free emission from optically-thin ionised gas $\left(\mathrm{S}_{v} \propto v^{-0.1}\right)$ if we are missing $\sim 34$ percent of the flux detected at $3.4-\mathrm{cm}$.

We also searched for $22 \mathrm{GHz}$ water maser emission across the filament. New masers were detected at six positions, as well as at the three previously known sites adjacent to IRS 1, IRS 3 and IRS 4. Figure 4 presents a cumulative spectrum from a single baseline showing the majority of the maser lines detected. Two groupings of lines are observed, the first centred at approximately $-115 \mathrm{~km} \mathrm{~s}^{-1}$ (with two components at -130 and $-100 \mathrm{~km} / \mathrm{s}^{-1}$ ) and the second centred at $-30 \mathrm{~km} \mathrm{~s}^{-1}$. The intense lines at -130 and $-100 \mathrm{~km} \mathrm{~s}^{-1}$ have peak amplitudes of $\sim 70$ and $\sim 900 \mathrm{Jy} /$ beam, respectively, and derive from maser sites towards the central $\mathrm{H}$ II region. Maser sites scattered across the 1.2-mm continuum filament contribute to the group of lines between -60 and $-10 \mathrm{~km} \mathrm{~s}^{-1}$. Figure 5 presents the maser spectra and illustrates the positions of individual maser sites superimposed on the $\mathrm{NH}_{3}(1,1)$ zeroth-moment image. Precise coordinates, intensities and velocities are noted in Table 3.

\subsection{Tidbinbilla results}

$\mathrm{NH}_{3}(1,1)$ and $(2,2)$ integrated intensity maps made using the Tidbinbilla 70-m telescope are presented in Fig. 6. Individual maps are centred on the same position as the ATCA fields, however, technical difficulties meant that we were unable to finish 


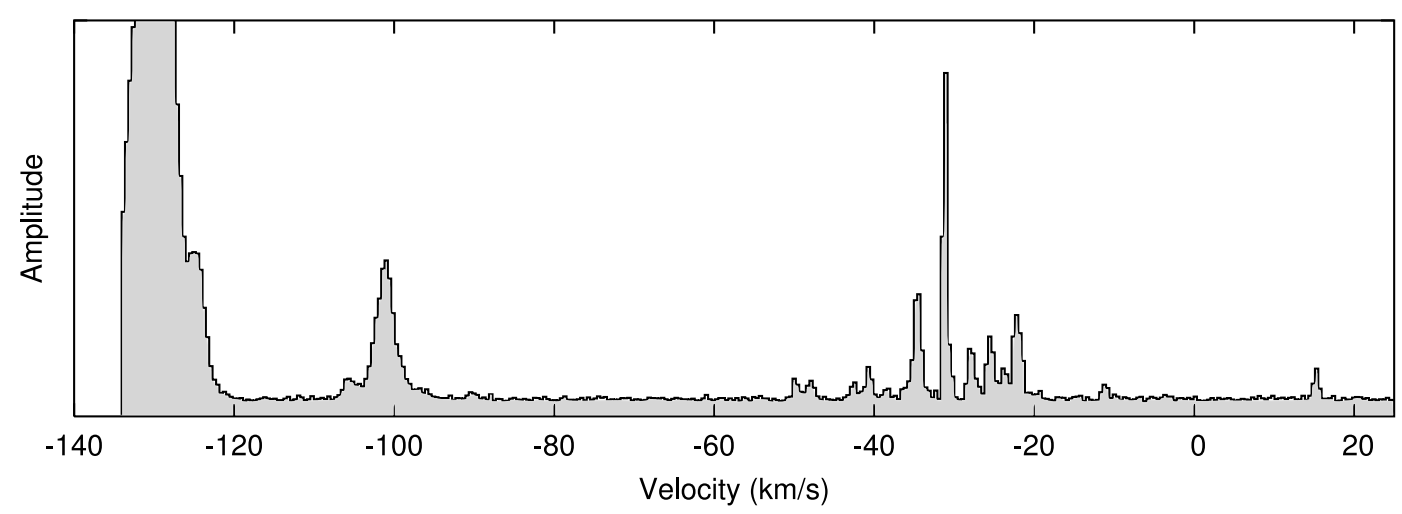

Fig. 4. Cumulative $\mathrm{H}_{2} \mathrm{O}$ maser spectrum from a single baseline showing lines from across the filament. The two broad maser lines at $-130 \mathrm{~km} \mathrm{~s}^{-1}$ and $-102 \mathrm{~km} \mathrm{~s}^{-1}$ derive from positions M9 and M5, respectively, towards the central $\mathrm{H}$ il region (see Table 3). The bulk of the maser lines concentrated near the systemic velocity at $-24 \mathrm{~km} \mathrm{~s}^{-1}$ are scattered across the filament. The units of amplitude are arbitrary.

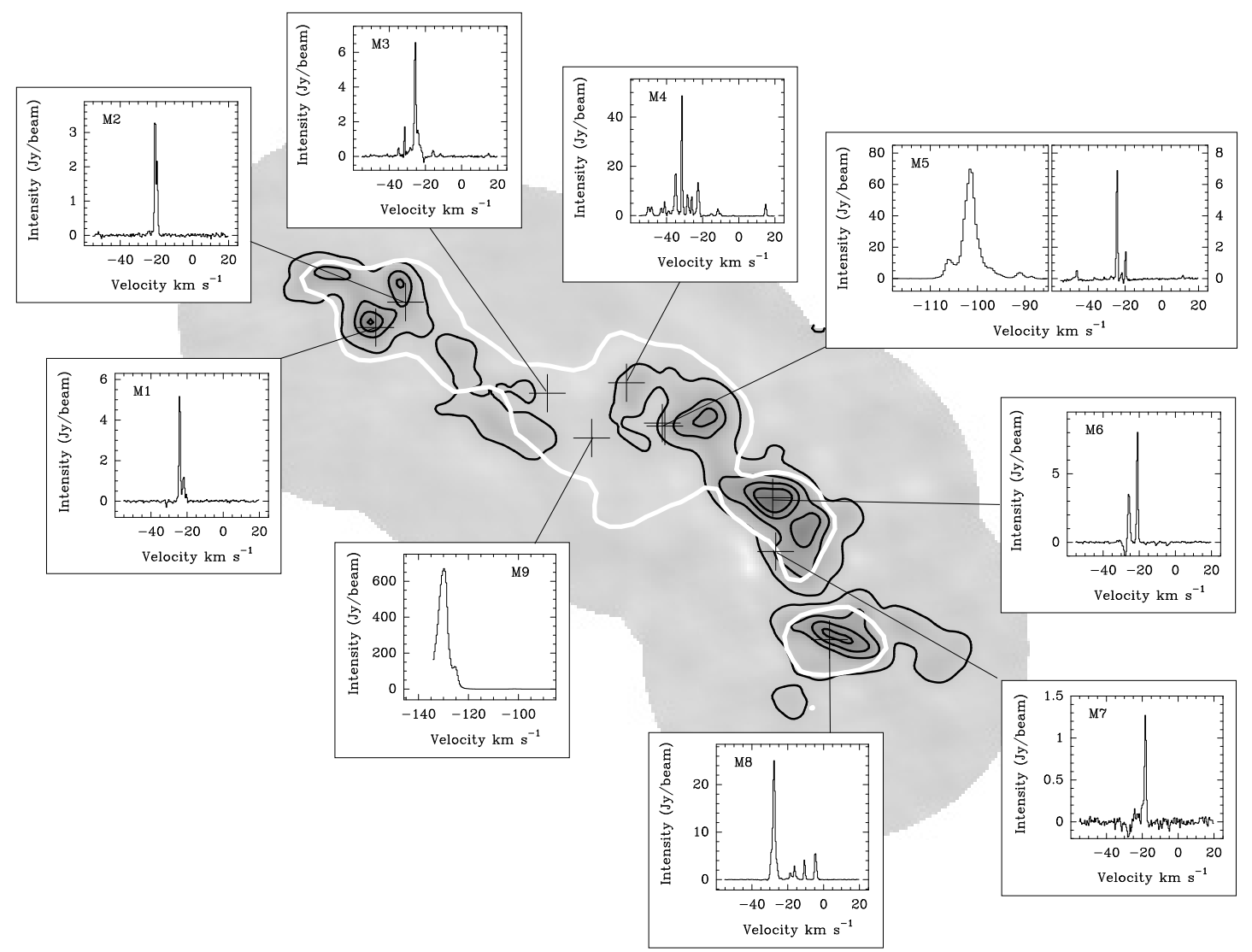

Fig. 5. Positions and spectra for the $22 \mathrm{GHz} \mathrm{H}_{2} \mathrm{O}$ masers detected in our ATCA EW367 array data. Six masers (M1, M2, M3, M6, M7 and M8) are new detections. Greyscale and black contours are the $\mathrm{NH}_{3}(1,1)$ integrated intensity map. The white line is the $\sim 3 \mathrm{Jy} /$ beam contour from of the 1.2-mm continuum map. Individual spectra are sampled from the peak maser pixel in the cleaned images of five ATCA fields.

Table 3. $22 \mathrm{GHz} \mathrm{H}_{2} \mathrm{O}$ masers in NGC 3576 .

\begin{tabular}{llccccc}
\hline \hline Maser & $\begin{array}{l}\text { RA } \\
(\mathrm{J} 2000)\end{array}$ & $\begin{array}{c}\text { Dec } \\
(\mathrm{J} 2000)\end{array}$ & $\begin{array}{c}\text { Velocity range } \\
\left(\mathrm{km} \mathrm{s}^{-1}\right)\end{array}$ & $\begin{array}{c}\text { Peak } V_{\text {LSR }} \\
\left(\mathrm{km} \mathrm{s}^{-1}\right)\end{array}$ & $\begin{array}{c}\text { Peak } \\
(\text { Jy/beam })\end{array}$ & Reference \\
\hline M1 & $11^{\mathrm{h}} 12^{\mathrm{m}} 16.72^{\mathrm{s}}$ & $-61^{\mathrm{d}} 17^{\mathrm{m}} 47.0^{\mathrm{s}}$ & -27 to -19 & -24.3 & 5.1 & New \\
M2 & $11^{\mathrm{h}} 12^{\mathrm{m}} 13.95^{\mathrm{s}}$ & $-61^{\mathrm{d}} 17^{\mathrm{m}} 30.0^{\mathrm{s}}$ & -22 to -18 & -20.7 & 3.3 & New \\
M3 & $11^{\mathrm{h}} 12^{\mathrm{m}} 00.76^{\mathrm{s}}$ & $-61^{\mathrm{d}} 18^{\mathrm{m}} 31.0^{\mathrm{s}}$ & -41 to +15 & -25.5 & 6.5 & New \\
M4 & $11^{\mathrm{h}} 11^{\mathrm{m}} 53.40^{\mathrm{s}}$ & $-61^{\mathrm{d}} 18^{\mathrm{m}} 24.0^{\mathrm{s}}$ & -51 to +17 & -31.4 & 46.5 & Caswell 2004 \\
M5 & $11^{\mathrm{h}} 11^{\mathrm{m}} 50.07^{\mathrm{s}}$ & $-61^{\mathrm{d}} 18^{\mathrm{m}} 51.0^{\mathrm{s}}$ & -110 to +12 & -101.5 & 69.3 & Caswell 2004 \\
M6 & $11^{\mathrm{h}} 11^{\mathrm{m}} 39.80^{\mathrm{s}}$ & $-61^{\mathrm{d}} 19^{\mathrm{m}} 41.0^{\mathrm{s}}$ & -27 to -20 & -20.9 & 7.8 & New \\
M7 & $11^{\mathrm{h}} 11^{\mathrm{m}} 39.52^{\mathrm{s}}$ & $-61^{\mathrm{d}} 20^{\mathrm{m}} 17.0^{\mathrm{s}}$ & -25 to -17 & -18.4 & 1.3 & New \\
M8 & $11^{\mathrm{h}} 11^{\mathrm{m}} 34.52^{\mathrm{s}}$ & $-61^{\mathrm{d}} 21^{\mathrm{m}} 16.0^{\mathrm{s}}$ & -31 to -3 & -27.7 & 24.8 & New \\
M9 & $11^{\mathrm{h}} 11^{\mathrm{m}} 56.64^{\mathrm{s}}$ & $-61^{\mathrm{d}} 19^{\mathrm{m}} 01.0^{\mathrm{s}}$ & $<-134$ to -121 & -129.9 & 670.5 & Caswell 2004 \\
\hline
\end{tabular}



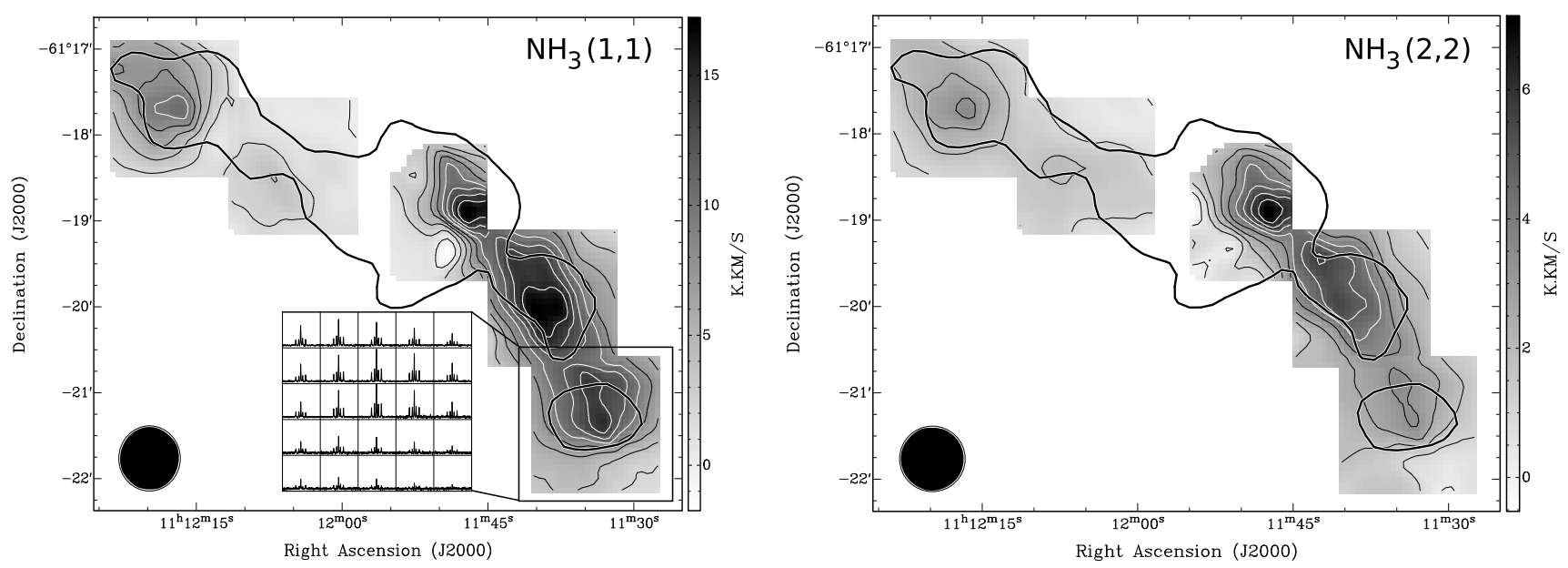

Fig. 6. $\mathrm{NH}_{3}(1,1)$ and $(2,2)$ integrated intensity maps made using the $70-\mathrm{m}$ Tidbinbilla telescope (greyscale and contours). Contours levels are at 10 percent intervals. The single thick black contour shows the extent of the $1.2 \mathrm{~mm}$ continuum emission (Hill et al. 2005). The grid inset into the $\mathrm{NH}_{3}(1,1)$ map illustrates the spectra which contributed to the map of the S5 field.

Table 4. ATCA $\mathrm{NH}_{3}$ percentage flux detected.

\begin{tabular}{lc}
\hline \hline Field & $\begin{array}{c}\text { Percentage flux } \\
\text { detected }\end{array}$ \\
\hline S1 & 94 \\
S2 & 78 \\
S3 & 80 \\
S4 & 91 \\
S5 & 70 \\
\hline
\end{tabular}

one column of field S3, covering the $\mathrm{H}$ II region. It is immediately evident that the morphology of the emission is similar to that seen in the the ATCA NH $3(1,1)$ data.

We initially intended to use data from the 70-m antenna to "fill in" the missing short-spacings in our ATCA data. For the merging to be successful, the emission in the single-dish dataset must be imaged out to its extremes. Unfortunately, there is still considerable flux at the edge of all fields and, due to time constraints and the experimental nature of the observations, we were unable to enlarge the maps. Instead, the Tidbinbilla data is used to estimate the fraction of missing flux due to extended emission in each of the ATCA fields. To do this we smoothed the final ATCA maps to the same resolution and pixel scale as the Tidbinbilla maps and compared the integrated intensities measured under the same aperture. The percentage ATCA/Tidbinbilla integrated intensity ratios are presented in Table 4. Calibration error on the Tidbinbilla data is approximately 30 percent and the measurements are consistent with less than 10 percent missing flux in the ATCA fields.

The estimates assume a beam-efficiency of 48 per cent for the 70-m telescope (Greenhill et al. 2003) and a (Jy/Beam)/K conversion factor of 0.951 .

\subsection{Mopra results}

Figure 7 presents integrated intensity maps and sample spectra for all six molecules detected by Mopra towards NGC 3576. The data have been smoothed to a velocity resolution of $0.4 \mathrm{~km} \mathrm{~s}^{-1}$ and have a spatial resolution of $40^{\prime \prime}$. Sample spectra presented alongside each map were extracted from the datacubes at the peak positions of the SIMBA clumps (labelled
S1-S5) identified in the 1.2-mm continuum map and marked by filled circles in Fig. 7.

As with $\mathrm{NH}_{3}$, the distribution of the other molecular lines closely follow the 1.2-mm emission except to the east of the central $\mathrm{H}$ II region. ${ }^{13} \mathrm{CO}(1-0)$ and $\mathrm{HCO}^{+}(1-0)$ trace more extended gas than the other lines, and their spectra appear moderately optically thick compared to their isotopologues, $\mathrm{C}^{18} \mathrm{O}(1-$ $0)$ and $\mathrm{H}^{13} \mathrm{CO}^{+}(1-0) . \mathrm{N}_{2} \mathrm{H}^{+}$and $\mathrm{CS}$ both trace dense gas, however $\mathrm{N}_{2} \mathrm{H}^{+}$exhibits optically thin line profiles $(\tau<0.1)$ at all positions while CS appears optically thick in places. The relative intensity of the emission between species varies significantly over the extent of the cloud, likely due to differences in the chemistry of the clumps. We discuss this possibility further in Sect. 5.3.

At position $\mathrm{S} 2$ the spectra of all species display three line components, offset in velocity by $\sim 4 \mathrm{~km} \mathrm{~s}^{-1}$. Spectra sampled at the other positions appear as a single line profile. These lines are not well fit by single Gaussians except at position S5 and are possibly composed of several blended features. Taking the optically thin $\mathrm{N}_{2} \mathrm{H}^{+}$as an example, the peak $V_{\text {lsr }}$ of the emission in the western arm (SIMBA clumps S3-S5) has a mean velocity of $-24.1 \pm 0.5 \mathrm{~km} \mathrm{~s}^{-1}$, while the eastern arm (SIMBA clumps S1 and S2) has a mean velocity of $-25.0 \pm$ $0.9 \mathrm{~km} \mathrm{~s}^{-1}$. No large velocity gradients are apparent, but the two arms are offset in velocity by $\sim 1 \mathrm{~km} \mathrm{~s}^{-1}$. The full-width halfmaximum $\mathrm{N}_{2} \mathrm{H}^{+}$linewidths ranges between approximately 1 and $3 \mathrm{~km} \mathrm{~s}^{-1}$ across the filament, with the highest values occurring adjacent to the $\mathrm{H}$ il region.

\section{Derived properties}

In this section we derive physical properties, such as temperature and density, from the molecular line data. The detailed methodology used in the calculations is presented in the appendix. We discuss the results in Sect. 5 where we analyse the morphology, kinematics, relative chemical abundances and star-forming content in detail.

\subsection{Physical properties from $\mathrm{NH}_{3}$}

The rotation-inversion transitions of $\mathrm{NH}_{3}$ have been used extensively in the literature to derive the kinetic temperature and column densities of molecular clouds (e.g., Ungerechts et al. 1986; 

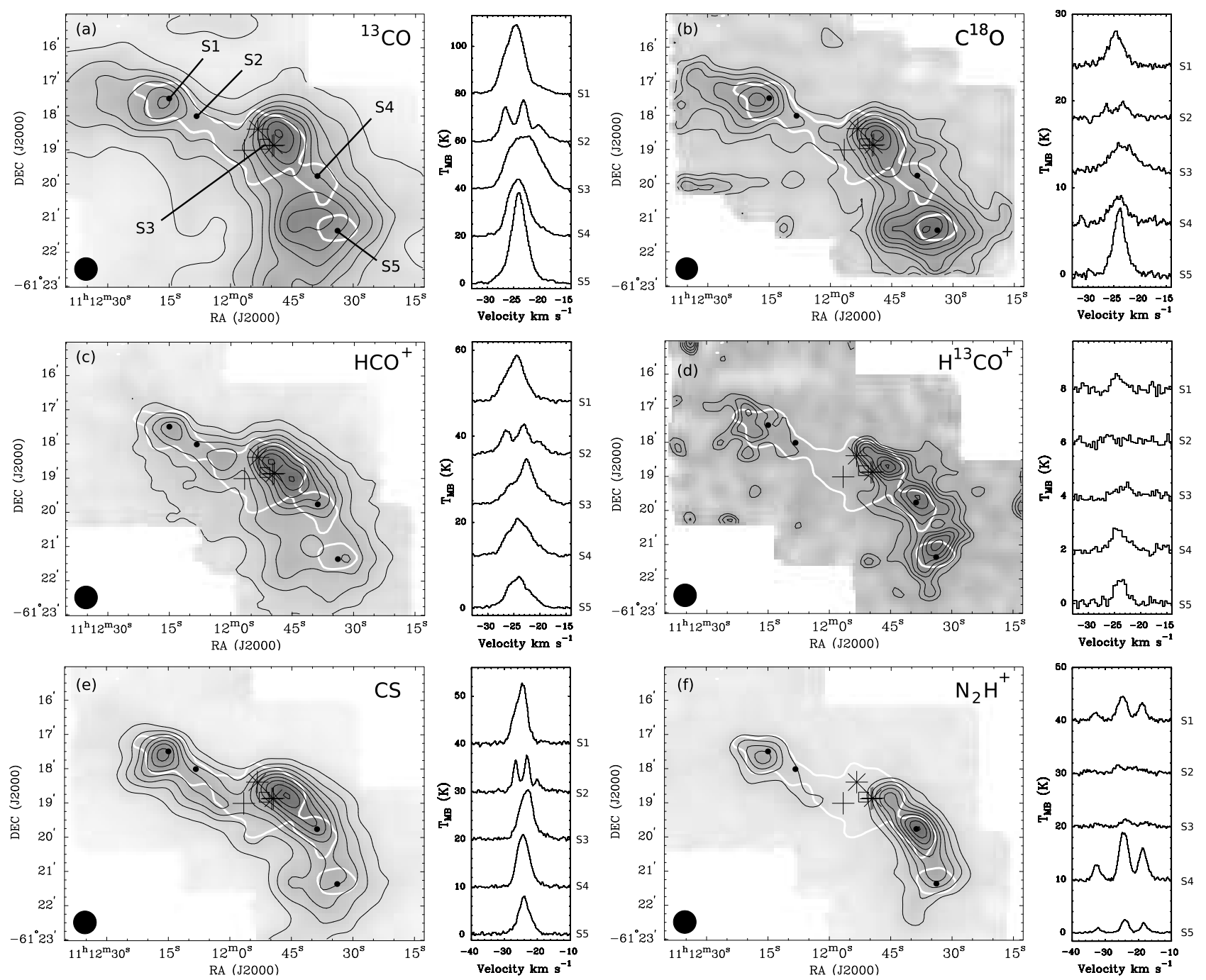

Fig. 7. Integrated intensity (zeroth-moment) maps of NGC 3576 in the six molecular transitions observed with the Mopra telescope (left panels). Sample spectra, extracted from the data cube at the positions S1-S5, are presented to the right of each map. ${ }^{13} \mathrm{CO}$ is generally optically thick and is a good tracer of the extended molecular envelope. $\mathrm{HCO}^{+}$and $\mathrm{H}^{13} \mathrm{CO}^{+}$trace similar gas densities to $\mathrm{CO}$, while $\mathrm{CS}$ and $\mathrm{N}_{2} \mathrm{H}^{+}$trace denser gas.

Cesaroni et al. 1992; Bourke et al. 1995). Due to its quantum mechanical properties, the $\mathrm{NH}_{3}$ inversion spectrum is split into multiple hyperfine components from whose brightness ratios the optical depth may be derived directly, hence removing a major assumption from the calculation of rotational temperatures.

\subsubsection{Kinetic temperature}

The modified rotational diagram method used to calculate rotational temperature $\left(T_{\text {rot }}\right)$ from $\mathrm{NH}_{3}$ has been described in detail by Ho et al. (1977), Ungerechts et al. (1980) and Townes et al. (1983). We provide a brief summary of the procedure in the appendix, specifically for the $\mathrm{NH}_{3}(1,1)$ and $(2,2)$ transitions. The molecular constants used in the calculations are collected in Table A.1.

Figure 8a presents a kinetic temperature map of NGC 3576 made using the $\mathrm{NH}_{3}(1,1)$ and $(2,2)$ data from the ATCA H75 array observations only. At a resolution of $\sim 23^{\prime \prime}$, the map reflects the beam averaged temperature of gas above a density of $n \approx 2 \times 10^{4} \mathrm{~cm}^{-3}$ (Swade 1989). Higher resolution observations would doubtlessly reveal regions with hotter or cooler temperatures than average. An obvious temperature gradient exists across parts of the filament. Figure $8 \mathrm{~b}$ plots kinetic temperature as a function of angular offset from the peak of the ionised emission. The data were sampled at 12 positions, corresponding roughly to the peaks of the $\mathrm{NH}_{3}$ clumps in Fig. 2. The median precision on the kinetic temperature values is approximately $2.4 \mathrm{~K}$ and stems from the uncertainties in the Gaussian fits. We find that the kinetic temperature is higher towards regions adjacent to the HII region. In the outlying positions the kinetic temperature is $\sim 12 \mathrm{~K}$, increasing to $\geq 30 \mathrm{~K}$ towards the centre. Some hot spots with temperatures above $40 \mathrm{~K}$ exist to the east of the $\mathrm{H}$ II region (between RAs of $11^{\mathrm{h}} 12^{\mathrm{m}} 00^{\mathrm{s}}$ and $11^{\mathrm{d}} 12^{\mathrm{m}} 15^{\mathrm{s}}$ ), but the signal-to-noise ratio in this part of the map is poor, hence the derived temperatures are more uncertain $(\sim 8 \mathrm{~K})$. Derived temperatures above $\sim 30 \mathrm{~K}$ have a large associated uncertainty because of assumptions made during the calculations (see Appendix A.1). However, below 20 K Tafalla et al. (2004) find that kinetic temperatures calculated under the same assumptions are accurate to better than 5 percent.

\subsection{2. $\mathrm{NH}_{3}$ column density and core mass}

Assuming local thermal equilibrium (LTE), the total column density of $\mathrm{NH}_{3}$ may be found directly from the integrated intensity of the $J, K=(1,1)$ line via Eq. (A.5) in the appendix. An 

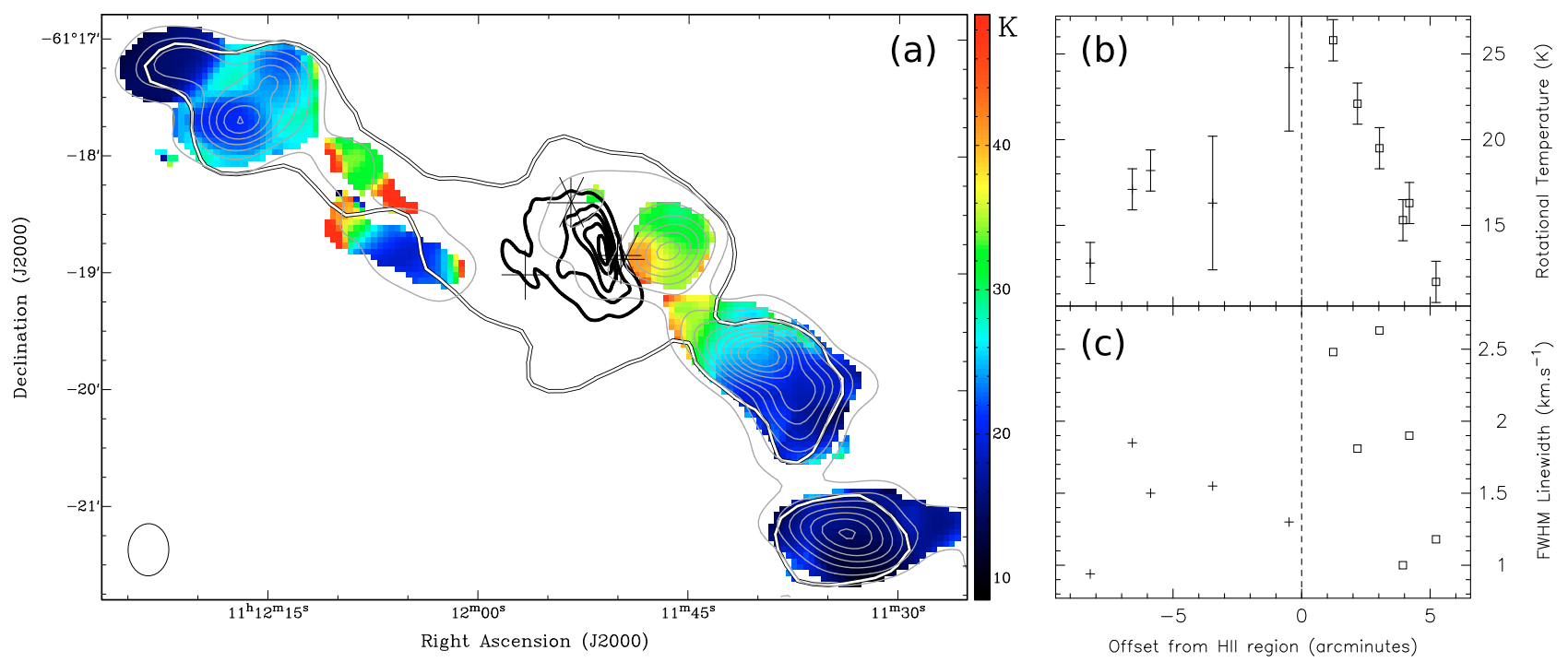

Fig. 8. a) Map of the kinetic temperature derived from the ratio of the $\mathrm{NH}_{3}(1,1)$ and $(2,2)$ lines. The beam size is $10.6^{\prime \prime} \times 11.9^{\prime \prime}$. Kinetic temperatures range from $>40 \mathrm{~K}$, in the centre of the map, to $10 \mathrm{~K}$ at the extremes; b) kinetic temperature; and $\mathbf{c}) \mathrm{NH}_{3}(1,1)$ linewidth as a function of angular offset from the peak of the HII region. Crosses represent positions eastwards of the $\mathrm{H}$ II region and squares positions to the west. A distinct temperature gradient is evident in the western arm of the filament (top-left).

estimate of the total mass of gas may then be determined from the column density assuming a constant relative abundance to $\mathrm{H}_{2}$.

In the $\sim 11^{\prime \prime}$ resolution $\mathrm{NH}_{3}(1,1)$ map we begin to resolve substructure within the $1.2-\mathrm{mm}$ clumps imaged by SIMBA. We have attempted to decompose the emission using the FELLWALKER $^{3}$ routine. FELLWALKER attempts to divide regions of emission into cores by searching for positive gradients in the datacube. The algorithm considers each pixel in turn above a lower brightness cutoff and "walks uphill", following the steepest ascent until an isolated local peak is reached. All pixels visited are assigned to the same core, which may already exist. Finally, cellular automata fill any holes in the cores and clean up the edges by replacing each cores' index with the the most common value occurring within a $3 \times 3 \times 3$ pixel cube. The signal/noise ratio was not constant across the mosaiced $\mathrm{NH}_{3}(1,1)$ map, so the FELLWALKER routine was run on the five SIMBA regions individually (using the mosaicked data-cube), starting at the same lower cutoff of $2-\sigma$, but slightly different tuning parameters. The results were inspected by eye and compared to the original datacube for consistency. Cores with fewer pixels than the area of the synthesised beam were omitted as unreliable detections.

Twenty-five believable cores were found, whose positions are plotted in Fig. 9a. The mass of $\mathrm{NH}_{3}$ in each core was calculated from the sum of the integrated intensities of the individual pixels in the core. We assumed excitation temperatures corresponding to those derived from the $23^{\prime \prime}$ resolution $\mathrm{H} 75$ data. The total core mass was then estimated assuming an abundance ratio $\left[\mathrm{NH}_{3}\right] /\left[\mathrm{H}_{2}\right]$ of $3.0 \times 10^{-8}$ (Wang et al. 2008), and a correction factor of 1.38 for the abundance of helium and heavier elements in the interstellar medium (Allen 1973). Table 5 presents the properties of the detected cores and the distribution of core masses is illustrated in Fig. 9b. In Sect. 5.2, below, we have calculated the $\mathrm{NH}_{3}$ abundance at different positions in the cloud

3 The FELLWALKER routine is part of the STARLINK package maintained at http://starlink. jach.hawaii.edu/ via a comparison to $450 \mu \mathrm{m}$ data. Column 11 presents the core masses corrected for these results.

Virial masses were calculated from the velocity width and average radius of the $\mathrm{NH}_{3}$ cores reported by FELLWALKER. Neglecting support from magnetic fields or internal heating sources, the virial mass of a simple spherical system is given by (MacLaren et al. 1988):

$M_{\text {vir }}=k r \Delta V^{2}$,

where $M_{\mathrm{vir}}$ is the core mass in $M_{\odot}, r$ is the deconvolved radius of the cloud in parsecs, $\Delta V$ is the $F W H M$ velocity width in $\mathrm{km} \mathrm{s}^{-1}$ and $k$ is an empirical constant depending on the assumed density distribution $\rho(r)$. For $\rho(r) \propto 1 / r^{-2}, k=126$ and for $\rho(r)=$ constant, $k=210$. Enhanced linewidths (e.g., due to optical depth effects or blending) will cause us to overestimate the virial mass and the values quoted here should be considered upper limits. We note that $\tau$ varies between 0.1 and 2.0, so line broadening due to optical depth effects is negligible. Columns 12 and 13 of Table 5 present the virial masses derived assuming constant and $1 / r^{2}$ density profiles, respectively.

\subsection{Physical properties from other molecules}

The molecules observed with Mopra towards NGC 3576 were chosen specifically to probe the physical conditions in different parts of the cloud. ${ }^{13} \mathrm{CO}\left(n_{\text {crit }} \approx 6 \times 10^{2} \mathrm{~cm}^{-3}\right.$, see Table A.1) and $\mathrm{C}^{18} \mathrm{O}$ emission traces the gas in the envelope surrounding the dense filament. $\mathrm{HCO}^{+}$and $\mathrm{H}^{13} \mathrm{CO}^{+}$have higher critical densities $\left(n_{\text {crit }} \approx 3 \times 10^{5} \mathrm{~cm}^{-3}\right.$ ) and so probe conditions at greater depths, but are also proven tracers of outflows and bulk gas motions (e.g. Rawlings et al. 2004). $\mathrm{CS}$ and $\mathrm{N}_{2} \mathrm{H}^{+}\left(n_{\text {crit }} \approx 2 \times 10^{5} \mathrm{~cm}^{-3}\right)$ have comparable critical densities but in practise have been shown to trace dense gas (e.g., Pirogov et al. 2003; Evans 1999 and references therein). $\mathrm{N}_{2} \mathrm{H}^{+}$especially, is considered to be useful indicator of the coldest $(\leq 10 \mathrm{~K})$ and most dense regions, where it is predicted to be the most abundant gas-phase ion (Caselli et al. 1995). This is due to the depletion of its main destruction partner, $\mathrm{CO}$, onto the dust grains. 

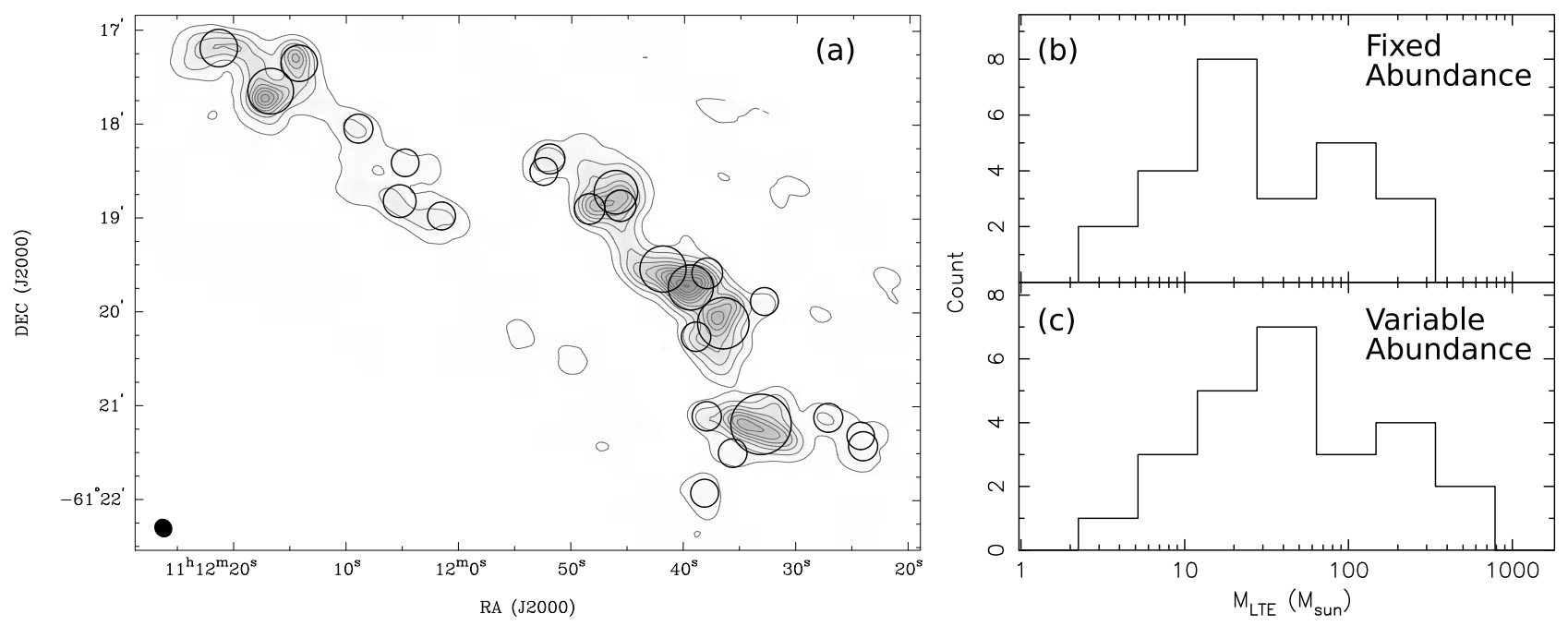

Fig. 9. a) The spatial distribution of cores detected in the $\mathrm{NH}_{3}(1,1)$ data cube using the FELLWALKER routine overplotted on the integrated intensity image. Circles mark the positions of the cores and the radii are scaled to the core mass, as reported in Table 5; b) histogram showing the distribution of core masses assuming a single value of $\left[\mathrm{NH}_{3}\right] /\left[\mathrm{H}_{2}\right]=3 \times 10^{-8} ; \mathbf{c}$ ) histogram showing the distribution of core masses corrected for the variable abundances calculated in Sect. 5.2.

Table 5. Properties of the $\mathrm{NH}_{3}(1,1)$ cores found using the FELLWALKER routine in the ATCA data.

\begin{tabular}{|c|c|c|c|c|c|c|c|c|c|c|c|c|c|c|}
\hline ID & $\begin{array}{l}\text { RA } \\
(\mathrm{J} 2000)\end{array}$ & $\begin{array}{l}\text { Dec } \\
(J 2000)\end{array}$ & $\begin{array}{c}\Delta \mathrm{RA}^{\alpha} \\
\left({ }^{\prime \prime}\right)\end{array}$ & $\begin{array}{c}\Delta \operatorname{Dec}^{\alpha} \\
\quad\left({ }^{\prime \prime}\right)\end{array}$ & $\begin{array}{c}\Delta V \\
\left(\mathrm{~km} \mathrm{~s}^{-1}\right)\end{array}$ & $\begin{array}{c}T_{\mathrm{MB}} \\
\mathrm{K}\end{array}$ & $\begin{array}{c}\sum T_{\mathrm{MB}} \mathrm{d} v \\
\left(\mathrm{~K} \mathrm{~km} \mathrm{~s}^{-1}\right)\end{array}$ & $\begin{array}{c}N_{\mathrm{NH}_{3}} \\
\left(\times 10^{15} \mathrm{~cm}^{-2}\right)\end{array}$ & $\begin{array}{c}M_{\mathrm{LTE}_{1}}{ }^{\beta} \\
\left(M_{\odot}\right)\end{array}$ & $\begin{array}{c}M_{\mathrm{LTE}_{2}}^{\gamma} \\
\left(M_{\odot}\right)\end{array}$ & $\begin{array}{l}M_{\mathrm{vir}_{1}} \delta \\
\left(M_{\odot}\right)\end{array}$ & $\begin{array}{l}M_{\mathrm{vir}_{2}}^{\dagger} \\
\left(M_{\odot}\right)\end{array}$ & $\begin{array}{c}T_{\text {kin }} \\
K\end{array}$ & $\tau$ \\
\hline 1 & $11: 12: 21.30$ & $-61: 17: 11.5$ & 12.4 & 4.9 & 1.3 & 20.4 & 2803.6 & 20.8 & 82 & 79 & 18 & 11 & 15 & 1.5 \\
\hline 2 & $11: 12: 16.70$ & $-61: 17: 39.0$ & 10.0 & 8.9 & 1.9 & 23.0 & 4989.6 & 36.7 & 144 & 166 & 41 & 25 & 20 & 0.7 \\
\hline 3 & $11: 12: 14.18$ & $-61: 17: 21.2$ & 6.4 & 8.4 & 2.4 & 18.5 & 2427.1 & 18.5 & 73 & 168 & 53 & 32 & 22 & 0.7 \\
\hline 4 & 11:12:08.91 & $-61: 18: 02.9$ & 6.8 & 6.1 & 1.2 & 6.1 & 485.6 & 3.9 & 15 & 15 & 11 & 7 & 33 & 0.1 \\
\hline 5 & 11:12:05.25 & $-61: 18: 49.3$ & 18.0 & 8.8 & 1.7 & 5.6 & 1538.2 & 11.3 & 44 & 44 & 48 & 29 & 19 & 0.1 \\
\hline 6 & $11: 12: 04.76$ & $-61: 18: 24.8$ & 7.1 & 1.6 & 0.8 & 8.5 & 131.7 & 1.2 & 5 & 5 & 3 & 2 & 47 & 0.1 \\
\hline 7 & 11:12:01.53 & $-61: 18: 58.7$ & 5.8 & 4.0 & 1.0 & 6.0 & 232.1 & 1.8 & 7 & 7 & 6 & 3 & 25 & 0.1 \\
\hline 8 & 11:11:52.45 & $-61: 18: 30.2$ & 4.2 & 3.7 & 0.6 & 23.9 & 185.2 & 1.6 & 6 & 47 & 2 & 1 & 31 & 0.1 \\
\hline 9 & 11:11:51.91 & $-61: 18: 22.2$ & 7.3 & 4.8 & 1.0 & 10.0 & 668.9 & 5.8 & 23 & 171 & 8 & 5 & 31 & 0.1 \\
\hline 10 & 11:11:48.39 & $-61: 18: 54.1$ & 2.8 & 3.7 & 1.2 & 28.4 & 809.4 & 7.0 & 27 & 91 & 6 & 3 & 40 & 0.1 \\
\hline 11 & 11:11:46.05 & $-61: 18: 43.4$ & 8.9 & 9.4 & 1.7 & 17.8 & 3990.0 & 32.5 & 128 & 426 & 32 & 19 & 33 & 0.1 \\
\hline 12 & 11:11:45.66 & $-61: 18: 52.0$ & 9.5 & 9.3 & 0.9 & 9.4 & 1051.4 & 8.6 & 34 & 112 & 10 & 6 & 35 & 0.1 \\
\hline 13 & 11:11:41.86 & $-61: 19: 32.4$ & 12.8 & 7.7 & 1.9 & 23.2 & 4935.5 & 37.8 & 148 & 62 & 44 & 27 & 26 & 0.4 \\
\hline 14 & 11:11:39.38 & $-61: 19: 44.2$ & 8.0 & 5.6 & 1.5 & 30.2 & 4757.6 & 35.5 & 139 & 59 & 20 & 12 & 23 & 0.7 \\
\hline 15 & 11:11:38.90 & $-61: 20: 15.6$ & 3.3 & 4.1 & 0.8 & 23.3 & 647.6 & 4.8 & 19 & 16 & 3 & 2 & 21 & 1.2 \\
\hline 16 & $11: 11: 38.11$ & $-61: 21: 55.7$ & 3.9 & 4.6 & 1.6 & 4.3 & 222.1 & 1.8 & 7 & 14 & 13 & 8 & - & 0.1 \\
\hline 17 & 11:11:37.94 & $-61: 21: 06.5$ & 1.3 & 2.8 & 0.8 & 85.9 & 595.2 & 4.5 & 18 & 35 & 1 & 1 & 15 & 1.2 \\
\hline 18 & 11:11:37.92 & $-61: 19: 35.1$ & 6.6 & 4.4 & 1.0 & 17.5 & 975.2 & 7.3 & 29 & 12 & 7 & 4 & 24 & 1.0 \\
\hline 19 & 11:11:36.49 & $-61: 20: 07.0$ & 7.6 & 12.3 & 1.7 & 25.9 & 6502.5 & 47.9 & 188 & 166 & 36 & 22 & 17 & 1.0 \\
\hline 20 & 11:11:35.62 & $-61: 21: 30.0$ & 7.9 & 2.6 & 0.6 & 20.5 & 422.7 & 3.2 & 12 & 25 & 3 & 2 & 15 & 2.2 \\
\hline 21 & 11:11:33.11 & $-61: 21: 11.6$ & 13.2 & 8.4 & 2.4 & 27.9 & 8117.8 & 65.7 & 258 & 516 & 78 & 47 & 15 & 1.9 \\
\hline 22 & 11:11:32.84 & $-61: 19: 53.0$ & 1.2 & 0.3 & 0.8 & 321.3 & 231.1 & 1.7 & 7 & 6 & 1 & 0 & 17 & 1.0 \\
\hline 23 & $11: 11: 27.13$ & $-61: 21: 07.3$ & 5.6 & 2.0 & 0.7 & 32.3 & 517.0 & 3.9 & 15 & 30 & 2 & 1 & 16 & 1.6 \\
\hline 24 & 11:11:24.25 & $-61: 21: 18.7$ & 5.2 & 6.2 & 0.8 & 4.4 & 109.9 & 0.9 & 3 & 7 & 5 & 3 & - & 0.1 \\
\hline 25 & 11:11:24.03 & $-61: 21: 25.3$ & 6.5 & 6.6 & 0.9 & 8.6 & 534.0 & 4.2 & 17 & 33 & 7 & 4 & - & 0.1 \\
\hline
\end{tabular}

${ }^{\alpha}$ Deconvolved full-width half-maximum $(F W H M)$ core size asumming an average beam $F W H M$ of $11.2^{\prime \prime} ;{ }^{\beta}$ LTE-mass derived from the integrated intensity of $\mathrm{NH}_{3}(1,1)$ assuming a relative abundance $\left[\mathrm{NH}_{3}\right] /\left[\mathrm{H}_{2}\right]=3 \times 10^{-8}{ }^{\gamma}{ }^{\gamma}$ Corrected LTE-mass, derived using the $\left[\mathrm{NH}_{3}\right] /\left[\mathrm{H}_{2}\right]$ abundance ratios calculated in Sect. 5.2 via a comparison between $\mathrm{NH}_{3}$ and $450 \mu \mathrm{m}$ p-ArTéMiS data; ${ }^{\delta} M_{\text {vir }}$ derived assuming a constant density profile; $M_{\text {vir }}$ derived assuming a $1 / \mathrm{r}^{2}$ density profile.

Assuming LTE conditions, we independently solved for the optical depth, excitation temperature and total column density of $\mathrm{CO}$ and $\mathrm{HCO}^{+}$via the procedure detailed in the Appendix A.3. Figure 10 presents the final column density maps towards NGC 3576. The ${ }^{13} \mathrm{CO}$ peak optical depth ranges from 0.1 to 4.4 and the excitation temperature from 6.4 to $36 \mathrm{~K}$. Peak $\mathrm{HCO}^{+}$optical depth ranges from 1.9 to 13.1 and excitation temperatures from 4.2 to $13 \mathrm{~K}$. We were unable to independently estimate the excitation temperatures of the $\mathrm{CS}$ and $\mathrm{N}_{2} \mathrm{H}^{+}$transitions and so adopted temperatures derived from the $\mathrm{NH}_{3}$ data. This is a reasonable assumption for $\mathrm{N}_{2} \mathrm{H}^{+}$as we expect both nitrogen bearing compounds to be intermixed in the gas phase. We were forced to assume that CS was optically thin, which may not be valid as some of the line profiles appear saturated. 
We calculated the total mass of the molecular cloud by assuming the following abundance ratios to $\mathrm{H}_{2}:\left[{ }^{13} \mathrm{CO}\right] /\left[\mathrm{H}_{2}\right]=$ $6.0 \times 10^{-6}$ (Goldsmith et al. 1997, assuming an abundance ratio of $\left[{ }^{12} \mathrm{CO}\right] /\left[\mathrm{H}_{2}\right]=45$ at a galactocentric radius of $8 \mathrm{kpc}-$ see Langer \& Penzias 1990), $\left[\mathrm{HCO}^{+}\right] /\left[\mathrm{H}_{2}\right]=2.0 \times 10^{-9}(\mathrm{Zhu}$ et al. 2007), $[\mathrm{CS}] /\left[\mathrm{H}_{2}\right]=1.1 \times 10^{-9}$ (Pirogov et al. 2007), and $\left[\mathrm{N}_{2} \mathrm{H}^{+}\right] /\left[\mathrm{H}_{2}\right]=5.0 \times 10^{-10}$ (Pirogov et al. 2007). The values for total cloud mass derived from the $\mathrm{CO}, \mathrm{HCO}^{+}$and $\mathrm{N}_{2} \mathrm{H}^{+}$observations are all approximately $8300 M_{\odot}$. The mass derived from CS is $12700 M_{\odot}$. These values assume calibration onto the Mopra "extended beam" temperature scale, which includes power received from the first sidelobe and is appropriate for data more extended than 80" (see Sect. 2.1 of Ladd et al. 2005).

\section{Analysis and discussion}

\section{1. $\mathrm{H}_{2} \mathrm{O}$ masers}

The three well-known water maser sites within the central H II region (labelled M4, M5 and M9 in Fig. 5) have been investigated by Caswell (2004), who summarises their properties. At the M4 site (G291.274-0.709) the $\mathrm{H}_{2} \mathrm{O}$ maser coexists with $6.67 \mathrm{GHz}$ $\mathrm{CH}_{3} \mathrm{OH}$ and $1.67 \mathrm{GHz} \mathrm{OH}$ masers. In the $\mathrm{H}_{2} \mathrm{O}$ maser spectrum we detect bright maser features ranging from $-37 \mathrm{~km} \mathrm{~s}^{-1}$ to $23 \mathrm{~km} \mathrm{~s}^{-1}$, in agreement with Caswell, however, we also detect weak $(<5 \mathrm{Jy})$ lines out to velocities of $-51 \mathrm{~km} \mathrm{~s}^{-1}$ and $+20 \mathrm{~km} \mathrm{~s}^{-1}$. The M5 site $(G 291.270-0.719)$ hosts a weak $\mathrm{CH}_{3} \mathrm{OH}$ maser at $-26 \mathrm{~km} \mathrm{~s}^{-1}$ and a bright $\mathrm{H}_{2} \mathrm{O}$ maser at a velocity of $-102 \mathrm{~km} \mathrm{~s}^{-1}$. First reported by Caswell et al. (1989), we find this $\mathrm{H}_{2} \mathrm{O}$ maser has an unusually broad linewidth $\left(2.9 \mathrm{~km} \mathrm{~s}^{-1}\right)$ and a peak flux density of $69 \mathrm{Jy}$, consistent with the original observations. The $V_{\mathrm{LSR}}$ of this spectral feature is reported to have become increasingly negative since its discovery in 1989 when it had a velocity of $-88 \mathrm{~km} \mathrm{~s}^{-1}$. This has been interpreted as being due to acceleration of the emitting gas, perhaps in a high velocity outflow (Caswell 2004; Caswell \& Phillips 2008). Alternatively, we suggest that the $-102 \mathrm{~km} \mathrm{~s}^{-1}$ line may be a new maser feature not associated with the component seen at $-88 \mathrm{~km} \mathrm{~s}^{-1}$, which may have been quenched in the intervening time. The final known site, M9 (G291.284-0.716), exhibits a single intense maser at a velocity of $-130 \mathrm{~km} \mathrm{~s}^{-1}$ and with a linewidth of $4.8 \mathrm{~km} \mathrm{~s}^{-1}$. We measure an intensity of $670 \mathrm{Jy}$ in contrast to the earlier value of $945 \mathrm{Jy}$ (Caswell et al. 1989), perhaps reflecting a real decrease in the intensity over the intervening time. We note that this maser feature falls on the edge of our bandpass, which may make the flux density measurement unreliable.

The six new maser sites reported here (M1, M2, M3, M6, M7 and M8) are located in the "arms" of the filament. As can be seen from Fig. 5 all sites are within a few arc-seconds of $\mathrm{NH}_{3}$ emission peaks. With the exception of M8, it is notable that the spectra of these new masers exhibit, at most, two bright features. These features have velocities close to the systemic velocity and intensities below $10 \mathrm{Jy}$. In contrast, the maser spectrum of M8 has four strong peaks spread over $\sim 35 \mathrm{~km} \mathrm{~s}^{-1}$, the brightest of which has an intensity of $25 \mathrm{Jy}$. The mid-infrared image of the host SIMBA clump (S5) also exhibits some nebulous emission, on the south-west edge (see Fig. 1a), while the dense molecular gas tracers (see Sects. 3.1, 3.2 and 3.3) show a "notch" in the emission at the same site. We interpret these features as evidence of a deeply embedded young stellar cluster evacuating an open-ended cavity.

\subsection{Core masses}

Core masses derived from $\mathrm{NH}_{3}$ rely on the assumption of a constant $\left[\mathrm{NH}_{3}\right] /\left[\mathrm{H}_{2}\right]$ abundance ratio, whose value may vary across the cloud. Independent estimates of the gas + dust-mass in NGC 3576 have been made by André et al. (2008), who mapped the $450 \mu \mathrm{m}$ thermal emission using the P-ArTéMiS ${ }^{4}$ bolometer camera on $\mathrm{APEX}^{5}$. With a beam size of $10^{\prime \prime}$ the $450 \mu \mathrm{m}$ map has a comparable resolution to our $\mathrm{NH}_{3}(1,1)$ observations and the morphology of the $450 \mu \mathrm{m}$ emission corresponds almost exactly to the integrated molecular emission.

We expanded the analysis of André et al. (2008) by decomposing the 2-D $450 \mu \mathrm{m}$ emission into cores using FELLWALKER and deriving their individual masses via the relation:

$$
M=\frac{S_{450} \mu \mathrm{m} D^{2}}{\kappa_{450} B_{v}\left(T_{\mathrm{d}}\right)_{450}},
$$

where $S_{450}$ is the measured flux density, $\kappa_{450}$ is the dust opacity per unit (gas + dust) mass column density at $450 \mu \mathrm{m}$, $B_{v}\left(T_{\mathrm{d}}\right)$ is the Planck function $B_{v}(T)$ at a dust temperature $T_{\mathrm{d}}$ and D is the distance to the source. We set $\kappa_{450}=0.04 \mathrm{~cm}^{2} \mathrm{~g}^{-1}$, a value generally adopted in sub-mm studies of pre-stellar cores (e.g. Motte et al. 1998, Ward-Thompson et al. 1999) and appropriate in regions of moderately high gas densities (around $10^{5} \mathrm{~cm}^{-3}-$ cf. Henning et al. 1995). For consistency, we set $T_{\mathrm{d}}$ to the kinetic temperatures derived from our $\mathrm{NH}_{3}$ data. A direct comparison between the masses derived from $450 \mu \mathrm{m}$ and $\mathrm{NH}_{3}$ emission integrated over the same apertures results in estimates of the average $\left[\mathrm{NH}_{3}\right] /\left[\mathrm{H}_{2}\right]$ abundance ratio in each core, assuming no depletion.

Eight equivalent cores were detected in the $450 \mu \mathrm{m}$ PArtéMiS and ATCA NH $\mathrm{NH}_{3}(1,1)$ integrated intensity maps, whose positions and masses are presented in Table 6. Mass values from $450 \mu \mathrm{m}$ data range from $\sim 89 M_{\odot}$ to $\sim 712 M_{\odot}$. Some $450 \mu \mathrm{m}$ cores encompass two or more $\mathrm{NH}_{3}(1,1)$ cores and in such cases we summed the masses of the components quoted in Col. 10 of Table 5 . Relative $\left[\mathrm{NH}_{3}\right] /\left[\mathrm{H}_{2}\right]$ abundance values range from $0.4 \times 10^{-8}$ to $7.1 \times 10^{-8}$ and are distinctly different in adjacent cores. We note that the error on the abundance values is approximately a factor of 3 , including the calibration uncertainty of the P-ArtéMiS data, assumptions about the dust emissivity and opacity, dust-to-gas ratio, temperature and relative angular size. The abundance differences may also be attributed to environmental factors. Cores S3-a and -b exhibit the lowest abundance values, but lie adjacent to the $\mathrm{H}$ II region. Here they may be exposed to the ultraviolet-radiation field from the central cluster, leading to enhanced destruction of large molecules. Core S4$a$, and to a much lesser extent S4-b, have elevated abundances of $\mathrm{NH}_{3}$. Interestingly, this SIMBA clump also has an enhanced abundance of $\mathrm{N}_{2} \mathrm{H}^{+}$, possibly due to depletion of $\mathrm{CO}$ onto the dust grain surface (see Sect. 5.3, below). In light of the varying abundance values we have attempted to correct the masses derived for the cores found in the $3-\mathrm{D} \mathrm{NH}_{3}$ data-cube. Where an equivalent $450 \mu \mathrm{m}$ core does not exist we revert to the canonical value of $3.0 \times 10^{-8}$ (Wang et al. 2008). These "corrected" values are presented in Col. 11 of Table 5.

Figure 11 compares the corrected LTE and virial masses for the $\mathrm{NH}_{3}$ cores in Table 5. The factor $f=M_{\text {corrected }} / M_{\text {vir }_{1}}$ measures the ratio of gravitational to kinetic energy. Values less than

\footnotetext{
4 See http://irfu.cea.fr/Sap/en/Phocea/Vie_des_labos/ Ast/ast_technique.php?id_ast $=2295$

5 Atacama Pathfinder Experiment http://www.apex-telescope. org/
} 

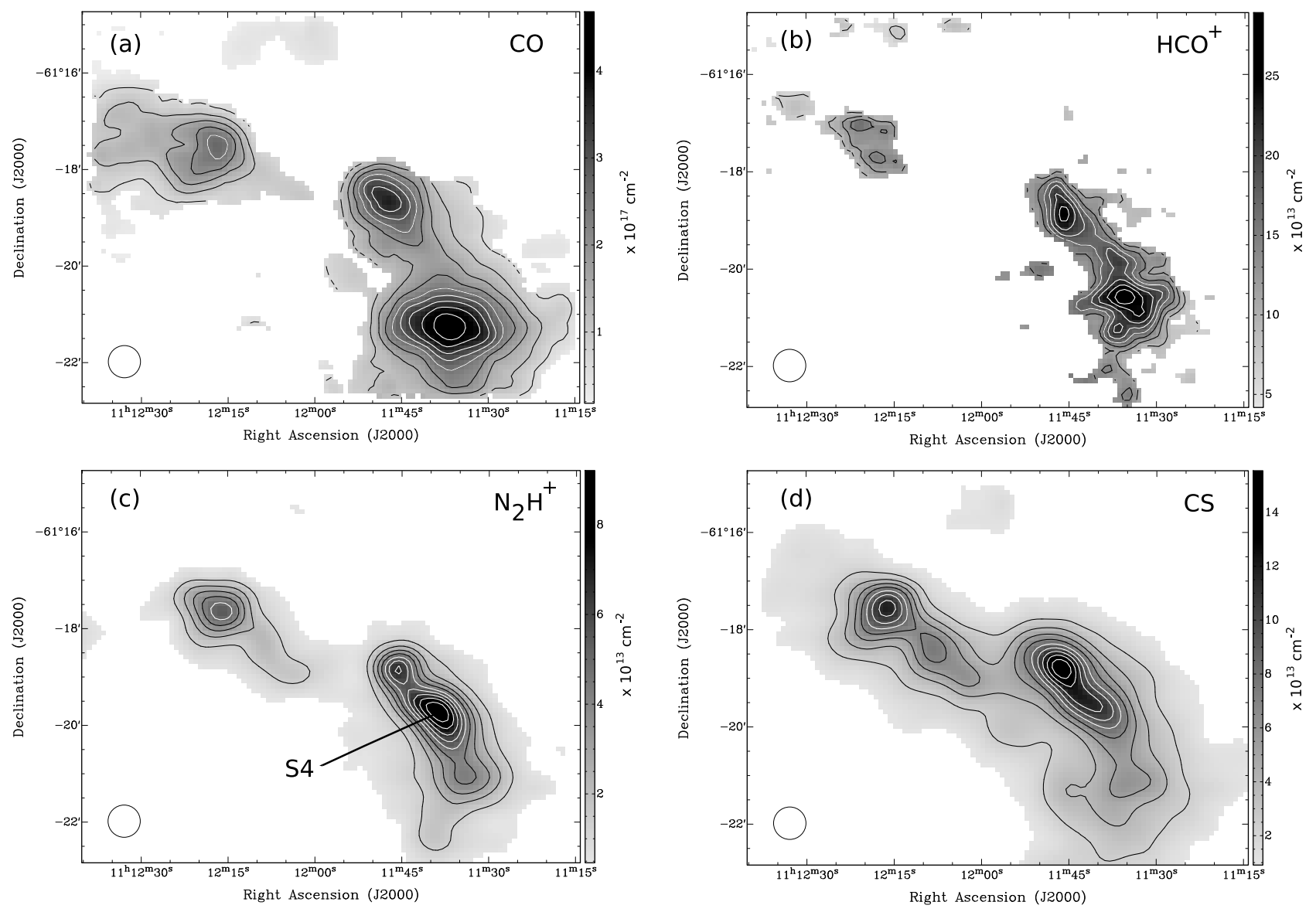

Fig. 10. Column density maps of the four species targeted by the Mopra telescope. Contours are set at 10 per cent intervals, beginning at 10 per cent, except in the $\mathrm{HCO}^{+}$column density map, where they start at 30 per cent of the peak value.

Table 6. Comparison of masses derived from p-ArTéMiS $450 \mu \mathrm{m}$ and ATCA NH $\mathrm{N}_{3}(1,1)$ emission.

\begin{tabular}{lccccccc}
\hline \hline $\begin{array}{l}\text { Clump } \\
\text { ID }\end{array}$ & $\begin{array}{c}\text { RA } \\
(\mathrm{J} 2000)\end{array}$ & $\begin{array}{c}\text { Dec } \\
(\mathrm{J} 2000)\end{array}$ & $\begin{array}{c}\text { Mass } \\
450 \mu \mathrm{m} \\
\left(M_{\odot}\right)\end{array}$ & $\begin{array}{c}\text { Mass } \\
\mathrm{NH}_{3} \text { LTE } \\
\left(M_{\odot}\right)\end{array}$ & $\begin{array}{c}{\left[\mathrm{NH}_{3}\right] /\left[\mathrm{H}_{2}\right]^{\alpha}} \\
\text { Abundance }\end{array}$ & $\begin{array}{c}\text { Average } \\
T_{\text {dust }}\end{array}$ & $\begin{array}{c}\text { Aliases } \\
\text { (André et al. 2008) }\end{array}$ \\
\hline S1-a & $11: 12: 21.09$ & $-61: 17: 09.50$ & 89 & 91 & $3.1 \times 10^{-8}$ & 15 & - \\
S1-b & $11: 12: 17.21$ & $-61: 17: 43.50$ & 161 & 140 & $2.6 \times 10^{-8}$ & 21 & S1-M1 \\
S1-c & $11: 12: 14.43$ & $-61: 17: 17.50$ & 193 & 82 & $1.3 \times 10^{-8}$ & 23 & S1-M2 \\
S3-a & $11: 11: 53.05$ & $-61: 18: 31.37$ & 357 & 51 & $0.4 \times 10^{-8}$ & 40 & S3-M4 \\
S3-b & $11: 11: 45.54$ & $-61: 18: 45.27$ & 682 & 211 & $0.9 \times 10^{-8}$ & 38 & S3-C3 \\
S4-a & $11: 11: 39.69$ & $-61: 19: 43.18$ & 161 & 383 & $7.1 \times 10^{-8}$ & 24 & S4-M6 \\
S4-b & $11: 11: 36.91$ & $-61: 20: 03.13$ & 247 & 279 & $3.4 \times 10^{-8}$ & 19 & - \\
S5 & $11: 11: 34.66$ & $-61: 21: 11.09$ & 712 & 339 & $1.4 \times 10^{-8}$ & 15 & S5-M8 \\
\hline
\end{tabular}

${ }^{\alpha}$ Relative abundance of $\mathrm{NH}_{3}$ to $\mathrm{H}_{2}$ via a direct comparison of clump masses derived from $\mathrm{NH}_{3}$ and $450 \mu$ m emission.

one indicate that the core may be a transient structure or confined by an over-pressured external medium, while a value of $f \sim 1$ suggests that the core is close to gravitational equilibrium. Cores with $f \gg 1$ are likely to be gravitationally unstable, meaning that internal magnetic fields are required to counteract the effects of self-gravity. We find that in all cases $f \geq 1$ (within errors) and seventeen cores have f-values between three and thirty. The magnetic field strength necessary to support each core may be calculated after Bot et al. (2007) via

$B^{2}-B_{0}^{2}=\frac{9}{10}\left(1-\frac{10}{9 f}\right) \frac{G M^{2} \mu_{0}}{R^{4} \pi}$, where $B_{0}=0.3 \mathrm{nT}$ is the ambient magnetic field strength in the Milky Way (Han et al. 2006), $\mathrm{R}$ is the core radius in metres and $\mathrm{M}$ is the core mass in kilograms. $\mathrm{G}$ and $\mu_{0}$ take their usual values in SI units. We calculate that field strengths ranging from $\sim 1 \mathrm{mG}$ to $\sim 40 \mathrm{mG}$, with a median of $\sim 4 \mathrm{mG}$, are required to balance self-gravity in addition to the turbulent pressure. By comparison, Curran \& Chrysostomou (2007) conducted polarimetry measurements of the magnetic field strengths in fourteen massive star forming regions and found values from $<0.1 \mathrm{mG}$ to $\sim 6 \mathrm{mG}$. Our values are higher on average, suggesting that some of our $\mathrm{NH}_{3}$ cores may indeed be collapsing. In Sect. 5.4 below we present evidence of collapse in the SIMBA clump S4. 


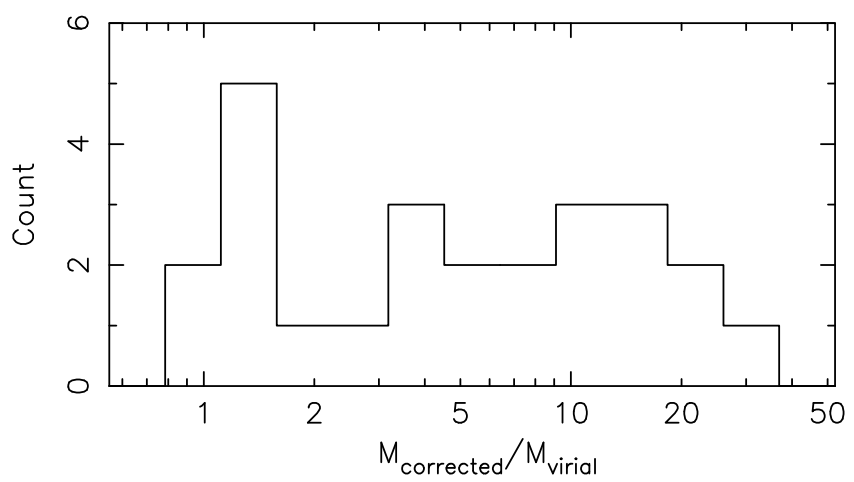

Fig. 11. Distributions of $M_{\text {corrected }} / M_{\text {vir }}$ for the 25 cores found in the $\mathrm{NH}_{3}$ data. The $M_{\text {corrected }}$ values have been derived from the $\mathrm{M}_{\mathrm{LTE}}$ masses scaled to the $450 \mu \mathrm{m}$ core masses. $M_{\mathrm{vir}}$ was calculated assuming a assuming a $\mathrm{r}^{-2}$ density profile. For a constant density profile virial masses would be 1.7 times larger. All cores have ratios $M_{\text {corrected }} / M_{\text {vir }} \geq 1$ and are at least consistent with being gravitationally bound. Cores with ratios much greater than one are likely to be gravitationally unstable and may be undergoing collapse unless supported by magnetic fields on the order of milli-Gauss

André et al. (2008) estimated the envelope mass of the dominant protostellar object in each $450 \mu \mathrm{m}$ core by scaling the mass corresponding to the peak flux density to a uniform diameter of $6000 \mathrm{AU}$, assuming a density profile $\rho(\mathrm{r}) \propto \mathrm{r}^{-2}$. The values range from 21 to $45 M_{\odot}$. They also estimated the bolometric luminosities by fitting the SIMBA, P-ArTéMiS and $\mathrm{MSX}^{6}$ data with a grid of spectral energy distribution models computed by Robitaille et al. $(2006,2007)$. When plotted on a $M_{\text {env }}$ versus $L_{\text {bol }}$ diagram (see Fig. 4 of André et al. 2008) the objects are bracketed by evolutionary tracks corresponding to final stellar masses of 15 to $50 M_{\odot}$, implying that the cores are undergoing high-mass star-formation. The relative positions of the cores on the diagram also indicates their relative evolutionary ages. Interestingly, the cores furthest from the $\mathrm{H}$ II region, at positions S1-b, S1-c, S4-a, and S5, are significantly less evolved than the adjacent cores at S3-a and S3-b. This evolutionary gradient is consistent with the hypothesis that the central $\mathrm{H}$ II region has triggered sequential star-formation in the filament.

\subsection{Chemistry}

Once the physical conditions of temperature, density and optical-depth have been determined, we can begin to examine the chemistry in NGC 3576. Ratios of column density provide beam-averaged measures of the relative abundances between two species. For key molecules this ratio is tied directly to the physical conditions and hence to the embedded star formation activity.

Figure 12 presents maps of the relative abundance of $\left[\mathrm{N}_{2} \mathrm{H}^{+}\right] /[\mathrm{CO}]$ and $\left[\mathrm{N}_{2} \mathrm{H}^{+}\right] /[\mathrm{CS}]$ made by taking the ratios of the column density maps. The abundance ratios are beam-averaged and assumes all of the gas is above the critical density for each species. Immediately clump $\mathrm{S} 4$ stands out in the $\left[\mathrm{N}_{2} \mathrm{H}^{+}\right] /[\mathrm{CO}]$ map, as it has an over-abundance of $\mathrm{N}_{2} \mathrm{H}^{+}$compared to the other SIMBA clumps. The difference is not as pronounced in the $\left[\mathrm{N}_{2} \mathrm{H}^{+}\right] /[\mathrm{CS}]$ map, however, we note the optical depth of

\footnotetext{
${ }^{6}$ Midcourse Space Experiment (MSX)

http://irsa.ipac.caltech.edu/applications/MSX/
}

CS is not known and hence there are significant uncertainties in the column density across the map. A comparison between the $\mathrm{N}_{2} \mathrm{H}^{+}$and $\mathrm{HCO}^{+}$column densities also reveals the same enhancement in clump S4, which we believe to be a real chemical difference. Because of the low signal-to-noise we do not show this map.

The abundance of $\mathrm{N}_{2} \mathrm{H}^{+}$is predicted to be enhanced in cold $(\leq 10 \mathrm{~K})$ or dense clumps. This is because its parent molecule $\mathrm{N}_{2}$ is one of the least affected by the condensation process and $\mathrm{N}_{2} \mathrm{H}^{+}$survives in the gas phase, at least for densities in the range $10^{5}-10^{6} \mathrm{~cm}^{-3}$. In such environments its main destruction partner $\mathrm{CO}$ depletes onto the grains and the abundance of $\mathrm{N}_{2} \mathrm{H}^{+}$consequently increases (Bergin et al. 2002). Interestingly, ${ }^{13} \mathrm{CO}$ and $\mathrm{C}^{18} \mathrm{O}$ emission are noticeably absent from clump $\mathrm{S} 4$, providing further confidence in this interpretation. Conditions such as these are found in starless cores, but particularly in cold collapsing clumps evolving towards forming stars. Clump S4 has an average kinetic temperature of $\sim 25 \mathrm{~K}$ according to our analysis of $\mathrm{NH}_{3}$ data. This seems high for an infrared dark cloud (see Fig. 1a) undergoing collapse, however, CO depletion may still occur at these temperatures if the density is high enough (Aikawa et al. 2001). In the following sub-section we will examine the evidence for inflowing gas motions in this clump.

\subsection{Morphology and kinematics}

We have examined the molecular emission for evidence of velocity gradients indicating bulk gas motions, expansion or contraction. Figure 13 presents position-velocity (PV) diagrams made using the ${ }^{13} \mathrm{CO}$ Mopra data and annotated on the inset integrated intensity map. Panel 1 was made using the $k$ shell tool ${ }^{7}$ and shows the azimuthly averaged intensity as a function of velocity and angular offset from position $11^{\mathrm{h}} 11^{\mathrm{m}} 58.0^{\mathrm{s}}-61^{\mathrm{d}} 18^{\mathrm{m}} 35.0^{\mathrm{s}}$ J2000. The ' $C$ ' shaped profile is a classic indicator of an expanding shell of gas, in this case centred approximately on the $\mathrm{H}$ II region. Towards the centre position (zero offset) the spatial pixels sample gas moving directly towards and away from the observer, hence the average spectrum peaks at the systemic velocity \pm the shell expansion velocity. At offsets approaching the shell radius the pixels sample gas moving perpendicular to the observer, hence the emission peaks at the systemic velocity $\left(-24 \mathrm{~km} \mathrm{~s}^{-1}\right)$. The clumpy shell seen here in ${ }^{13} \mathrm{CO}$ is approximately 1 arcminute in diameter and encompasses the free-free emission from the $\mathrm{H}$ II region. We see exactly the same $\mathrm{C}$-shape in $\mathrm{C}^{18} \mathrm{O}$, although the plot is noisier.

Panels 2-4 present the PV-slices indicated on the inset ${ }^{13} \mathrm{CO}$ moment map (top-left). The first cut, (centred at $11^{\mathrm{h}} 11^{\mathrm{m}} 09.90^{\mathrm{s}},-61^{\mathrm{d}} 15^{\mathrm{m}} 45.6^{\mathrm{s}} \mathrm{J} 2000$ and at a position angle $\Theta=$ $244.5^{\circ}$ ) is a longitudinal slice through the eastern arm of the filament and reveals a forked cross-section: two tenuous tentacles of emission merge into the eastern-most SIMBA clump (S1). $\mathrm{PV}$-slices through the dense gas tracers $\left(\mathrm{NH}_{3}\right.$ and $\left.\mathrm{CS}\right)$ show the same structure, meaning that this morphology is not due to optical depth or chemical effects. Ionised gas from the $\mathrm{H}$ II region overlays this part of the eastern arm, likely inter-penetrating the molecular emission. Combined with the existence of an expanding shell and a temperature gradient, we conclude that the $\mathrm{H}$ II region is expanding eastwards, and has played a significant role in sculpting these features.

\footnotetext{
${ }^{7}$ kshell is part of the KARMA suite of analysis software available from the ATNF at http://www.atnf.csiro.au/computing/ software/
} 

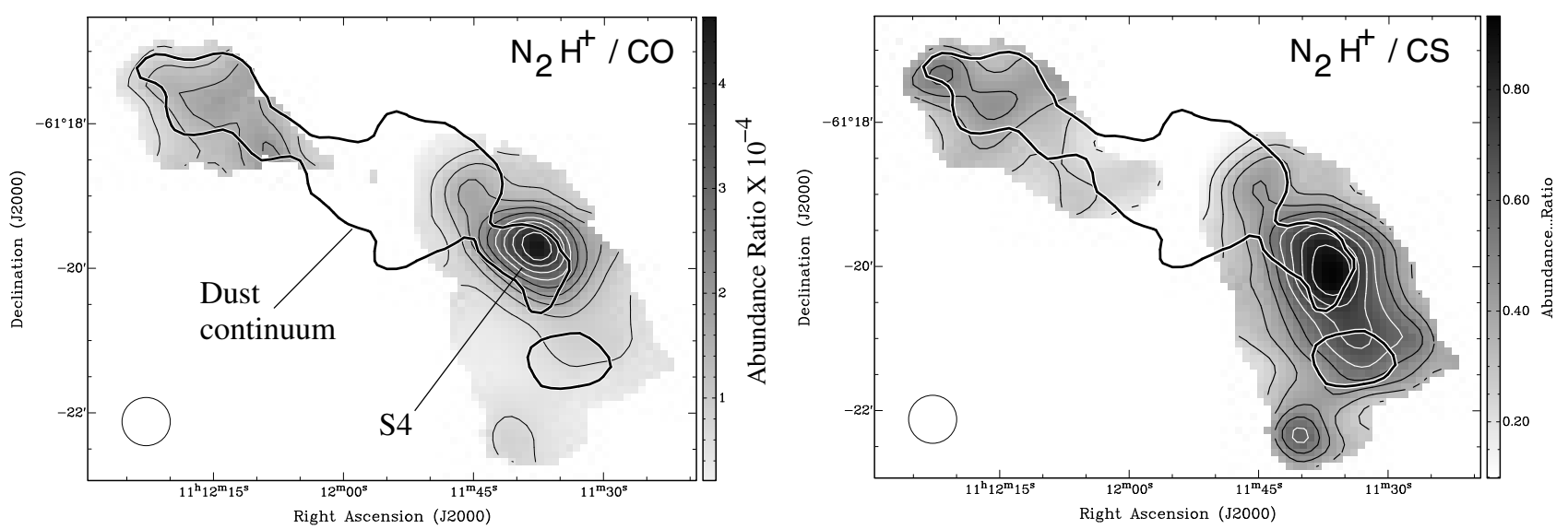

Fig. 12. Maps of the relative abundance of $\left[\mathrm{N}_{2} \mathrm{H}^{+}\right] /[\mathrm{CO}]$ (left) and $\left[\mathrm{N}_{2} \mathrm{H}^{+}\right] /[\mathrm{CS}]$ (right) made from individual column density maps. Greyscale and contours are set at 10 per cent intervals, starting at 30 per cent of peak. The $\mathrm{N}_{2} \mathrm{H}^{+}$abundance is significantly enhanced towards clump S4. This difference is especially pronounced in the $\left[\mathrm{N}_{2} \mathrm{H}^{+}\right] /[\mathrm{CO}]$ map but is not as clear when comparing $\mathrm{N}_{2} \mathrm{H}^{+}$and CS. We note that the column density of CS is the least well known of the three species.
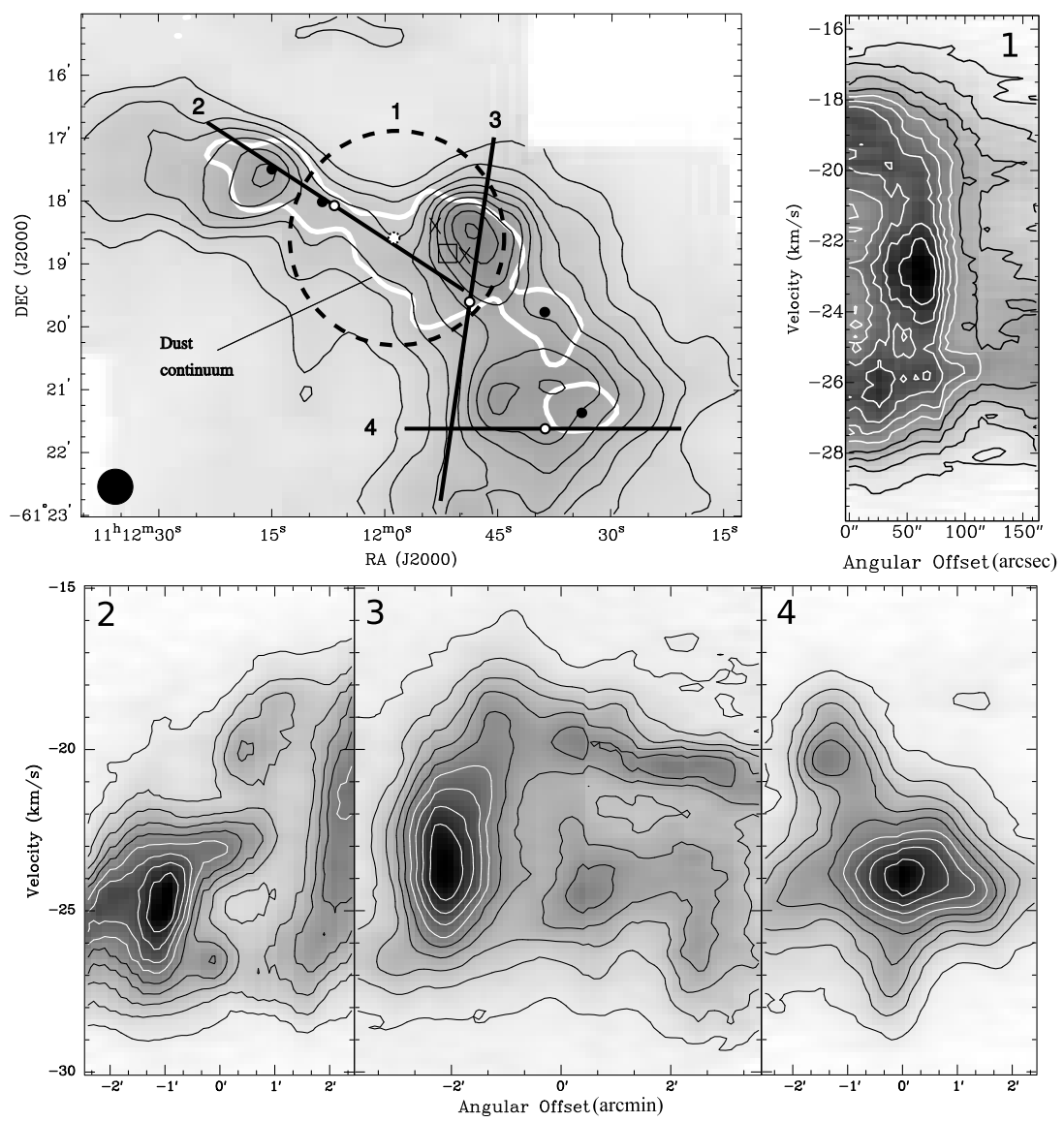

Fig. 13. Position-velocity cuts through the ${ }^{13} \mathrm{CO}$ data-cube. The top-left panel is an integrated intensity map of ${ }^{13} \mathrm{CO}$ showing the orientations and positions of the PV-slices presented in panels 1-4. Centre positions of the slices are marked with black and white filled circles. Panel 1 shows the azimuthly averaged intensity as a function of velocity and angular offset from position $11^{\mathrm{h}} 11^{\mathrm{m}} 58.0^{\mathrm{s}}-61^{\mathrm{d}} 18^{\mathrm{m}} 35.0^{\mathrm{s}} \mathrm{J} 2000$. The "C" shaped profile is indicative of an expanding shell of emitting gas. Panels 2, 3 and 4 present PV-slices along the axes of emission and in cross-section through the western arm, respectively. Several distinct filamentary structures are evident, merging into the bright emission adjacent to the $\mathrm{H}$ II region.

The second cut $\left(11^{\mathrm{h}} 11^{\mathrm{m}} 48.60^{\mathrm{s}},-61^{\mathrm{d}} 20^{\mathrm{m}} 57.6^{\mathrm{s}} \mathrm{J} 2000, \Theta=\right.$ $174^{\circ}$ ) follows the line of emission evident in Fig. 13 at $-20 \mathrm{~km} \mathrm{~s}^{-1}$. It is clear from the PV-diagram that two velocity components exist, separated by $\sim 4 \mathrm{~km} \mathrm{~s}^{-1}$. Both appear to merge into the strong emission immediately west of the $\mathrm{H}$ II region.
These two velocity features are clearly visible in the third cut $\left(11^{\mathrm{h}} 11^{\mathrm{m}} 39.50^{\mathrm{s}},-61^{\mathrm{h}} 21^{\mathrm{m}} 46.9^{\mathrm{s}} \mathrm{J} 2000, \Theta=268^{\circ}\right)$, which presents the velocity structure of the western arm in cross-section. The bulk of the emission in this arm derives from the component at $-24 \mathrm{~km} \mathrm{~s}^{-1}$, while the component at $-20 \mathrm{~km} \mathrm{~s}^{-1}$ is not seen in 

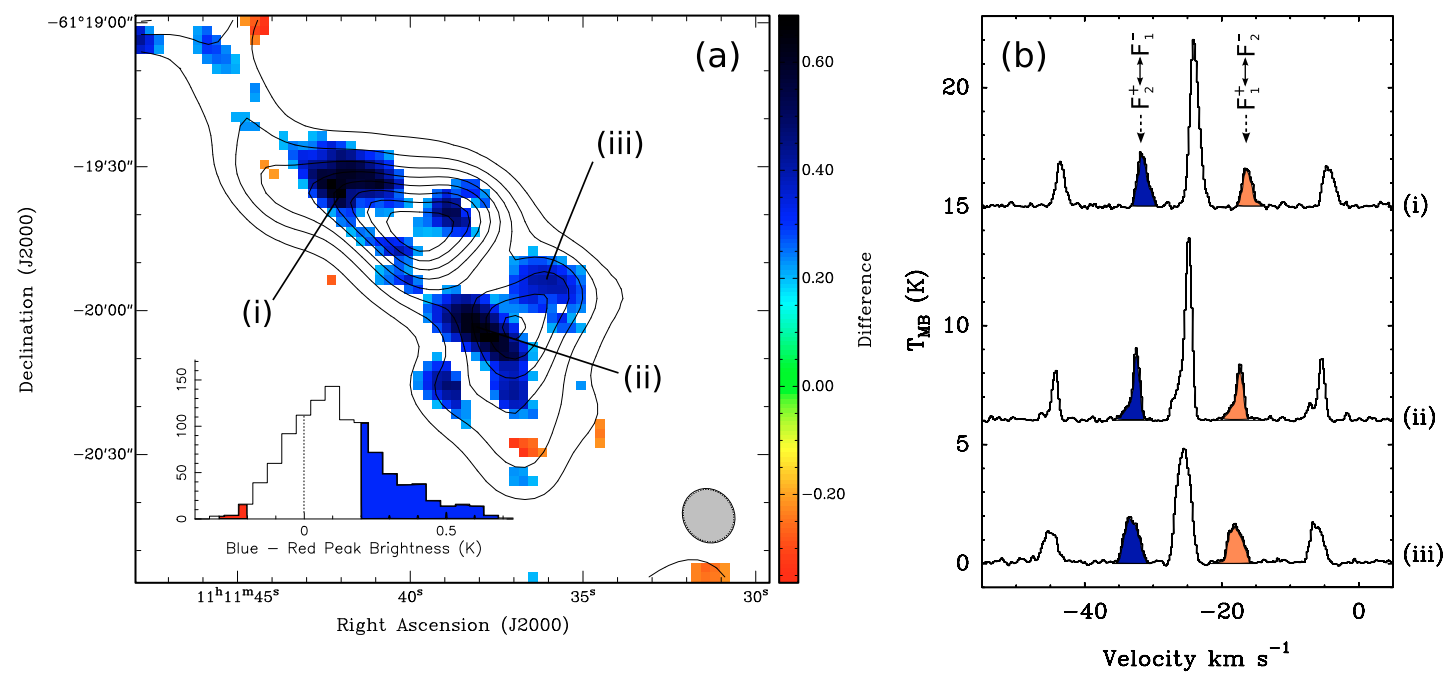

Fig. 14. a) Map of the difference between the peak intensities of the $\mathrm{F}_{2}^{+} \leftrightarrow \mathrm{F}_{1}^{-}$(blue) minus $\mathrm{F}_{1}^{+} \leftrightarrow \mathrm{F}_{2}^{-}$(red) inner satellite lines of $\mathrm{NH}_{3}(1,1)$ for SIMBA clump S4, where $\mathrm{F}$ is the total angular momentum quantum number (see Fig. 1 in Rydbeck et al. 1977). Only spectra with differences greater than $0.2 \mathrm{~K}(\sim 3-\sigma)$ are plotted as colourscale, while contours represent the integrated intensity map. The inset histogram shows the distribution of difference values over the whole of the clump and is clearly dominated by significant numbers of blue-skewed spectra. (b) Sample spectra from the positions marked i, ii and iii on the difference map.

the dense gas tracers $\mathrm{NH}_{3}, \mathrm{~N}_{2} \mathrm{H}^{+}$or $\mathrm{CS}$. Considering the dense gas only, we see the peak $\mathrm{V}_{\text {lsr }}$ of the filament is approximately constant between -24 to $-25 \mathrm{~km} \mathrm{~s}^{-1}$ over its length. No large opposing velocity gradients or discontinuities are evident and small variations may be explained by optical depth effects or motions on small scales $\left(<30^{\prime \prime}\right)$. We see no evidence for large scale flows of gas, at least in the $40^{\prime \prime}$ resolution Mopra data, and conclude that the majority of the emission derives from the same cloud and is not merely a projection or line-of-sight effect. The velocity structure in the $11^{\prime \prime}$ resolution $\mathrm{NH}_{3}$ data is complex within the bounds of each SIMBA clump and will be analysed in detail in a separate paper.

We are interested in probing for bulk gas motions in clump $\mathrm{S} 4$, where we find an over-abundance of $\mathrm{N}_{2} \mathrm{H}^{+}$, common in collapsing clumps. Asymmetric spectra towards star forming regions are often interpreted as indicators of inward or outward motions. In the case of $\mathrm{NH}_{3}(1,1)$ asymmetries may be produced by a combination of non-LTE effects and bulk motions. Park (2001) has modelled $\mathrm{NH}_{3}(1,1)$ spectra over a wide range of physical conditions, predicting that asymmetries between the inner satellite lines indicates inward or outward flows of gas. On the other hand, asymmetries between the outer satellites are indicative of selective radiative trapping and non-LTE excitation (Stutzki \& Winnewisser 1985). The amount by which both effects skew the profiles increases with optical depth. Figure 14a presents a map showing the difference between the blue minus red peak intensities of the inner satellite $\mathrm{NH}_{3}(1,1)$ lines, for SIMBA clump $\mathrm{S} 4$. Only pixels with absolute differences greater than $0.2 \mathrm{~K}(\sim 3-\sigma$ above the spectral noise $)$ have been plotted. Asymmetries on the order of $\sim 10$ percent are observed over distinct regions within the SIMBA clump. We note that there are few pixels with significant red-skewed spectra (values less than $-0.2 \mathrm{~K}$ in Fig. 14a) and the map is dominated by regions of blueskewed spectra which cover angular areas greater than the beam. Sample spectra from the three largest regions are presented in Fig. 14b. The optical depth of $\mathrm{NH}_{3}(1,1)$ in this clump ranges between $0.4 \leq \tau \leq 1.0$ and we do not expect large asymmetries under these conditions, hence, we believe the differences are significant and tentatively imply inward gas motions.

\section{5. $H$ ॥l region - cloud interaction}

It is clear from the temperature map presented in Fig. 8 that the central star-forming complex is heating the gas in its immediate surroundings. The heating is gradual on the western side of the filament and the temperature decreases linearly towards the extremes. The gradient in temperature strongly suggests that the HII region is embedded within the filament and is not merely a line of sight projection. The temperature fluctuates between $\sim 15$ and $\sim 30 \mathrm{~K}$ in the $\mathrm{S} 2$ clump, immediately east of the $\mathrm{H}$ II region's peak. Diffuse free-free emission is observed to extend into this region and the molecular gas has a clumpy distribution and is less evenly heated. It is interesting to note that there is a weak linewidth gradient in the western arm also, as plotted in Fig. 8c. This may reflect the turbulent energy injected into the cloud via shocks driven by the expanding ionised gas in the $\mathrm{H}$ II region. Clear evidence for this continuing expansion is seen in the ${ }^{13} \mathrm{CO}$ data as an expanding shell of molecular gas surrounding the free-free emission.

\subsection{Infrared excess stars}

Previous studies have focused on the infrared properties of embedded stars in NGC 3576. Emission in the $K(2.2 \mu \mathrm{m})$ and $L$ (3.6 $\mu \mathrm{m})$ bands, which cannot be accounted for by interstellar reddening, has been interpreted as the signature of thermal emission from a dusty disk. Maercker et al. (2006) imaged the region in $J(1.3 \mu \mathrm{m}), H(1.7 \mu \mathrm{m}), K$ and $L$, and found 113 stars with infrared excess. Their distribution is plotted in Fig. 15 over the ${ }^{13} \mathrm{CO}(1-0)$ integrated intensity map. Foreground stars have been filtered from the list. A dense cluster of IR-excess stars is centred on the Giant HII region, as reported in work by Persi et al. (1994) using Las Campanas Observatory. It is clear that the distribution of IR-excess stars is anti-correlated with the molecular gas in the dense arms of the filament. The high extinction in this area likely makes it impossible to detect even bright IR-excess sources behind or embedded in the filament. This suggests that the clumps are not associated with star-formation at an advanced stage of development. If young stars are present in the filament, 


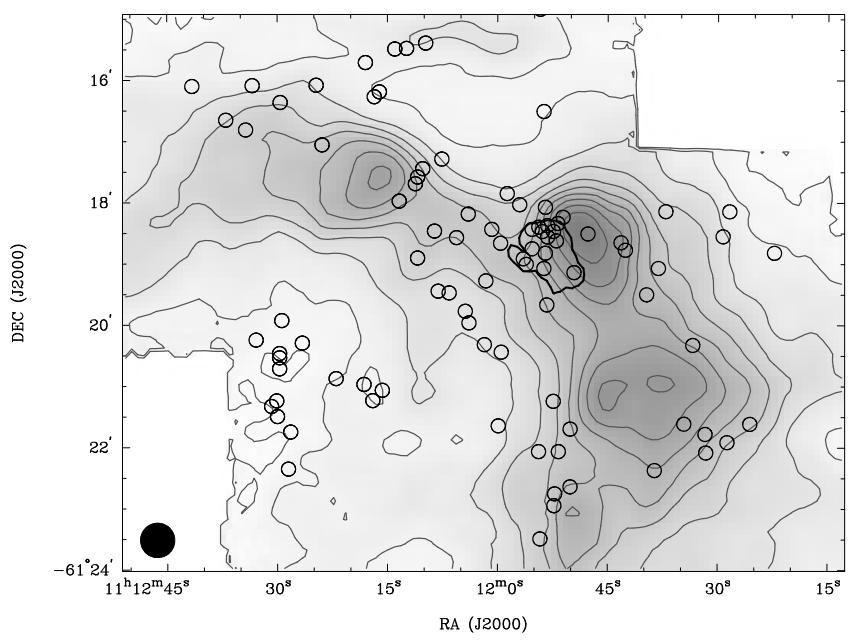

Fig. 15. The distribution of stars with infrared excess determined by Maercker et al. (2006), plotted over the molecular gas traced by ${ }^{13} \mathrm{CO}(1-0)$ emission.

they are probably at such a young age that they are still deeply embedded and not yet visible in the near-infrared.

\subsection{Triggered star formation in NGC 3576?}

Our observations provide an interesting glimpse into star formation activity within NGC 3576. It is clear that the $\mathrm{H}$ II region is embedded within the dusty filament and is exerting a powerful influence on its immediate surroundings. We have investigated whether the $\mathrm{H}$ in region expansion into the ambient molecular cloud is consistent with triggering the formation of high-mass stars along the dusty filament.

By comparing the morphology of the $\mathrm{H}$ II region (traced by the $23 \mathrm{GHz}$ continuum emission) to the dense molecular gas (traced by $\mathrm{NH}_{3}, \mathrm{CS}$ and $\mathrm{N}_{2} \mathrm{H}^{+}$) and thermal dust emission (traced by $1.2-\mathrm{mm}$ and $450 \mu \mathrm{m}$ continuum), we see that the young star cluster at the heart of the complex has destroyed or dispersed much of the gas in the central clump. There is also clear evidence for an expanding shell in the ${ }^{13} \mathrm{CO}$ and $\mathrm{C}^{18} \mathrm{O}$ data, centred on the $\mathrm{H}$ iI region.

To the east of the $\mathrm{H}$ II region, molecular gas is sparse and does not follow the morphology of the dust, while at $3.4-\mathrm{cm}$ wavelengths the ionised gas is observed to extend throughout most of field S2 (see Fig. 1c). In contrast, the western edge of the free-free continuum emission is observed to have a well defined boundary. We find from our molecular-line observations that this edge coincides with a dense molecular clump, implying that the $\mathrm{H}$ II region is pressure-confined on this side. At the same time, the $\mathrm{H}$ II region appears to be heating the arms of the filament and the western arm exhibits a clear temperature gradient, peaking adjacent to the $\mathrm{H}$ II region. In the eastern arm, the clumpy nature of the gas gives rise to "hot spots" with higher temperatures, consistent with our "dispersal" interpretation. The gradients in temperature strongly suggest that the $\mathrm{H}$ II region is embedded within the filament. Some of the structures visible in the eastern arm are reminiscent of the "elephant's trunks" pillars in the Eagle Nebula (White et al. 1999).

Besides the heating evidence, there is also evidence for kinematical interaction between the $\mathrm{H}$ II region and the ambient molecular cloud. We do see evidence that the $\mathrm{H}$ II region is expanding into the eastern arm, sweeping up a clumpy shell of gas.
Such an expansion is expected in the collect and collapse scenario for triggered star formation (see Sect. 1). Multiple velocity components have been identified within the arms of the filament (see Fig. 13). Velocity gradients and dislocations may be the archaeological remains of cloud-cloud collisions or quickly collapsing elongated clumps (c.f. Peretto et al. 2006). However, when considering only the dense gas we see that the velocity structure of the gas is smooth, confined to a narrow range and does not support either interpretation, at least at the 40 "scale of the Mopra data.

Different diagnostics have been employed to identify signatures of early phases of star formation in the filaments and gas clumps. Water masers have always been associated with star formation (see Beuther et al. 2002 for a summary). That we detect water-maser emission towards the arms of the filament confirms the presence of shocks and hence that stars are forming within the molecular gas. However, unlike $6.67 \mathrm{GHz} \mathrm{CH}_{3} \mathrm{OH}$ masers, $22 \mathrm{GHz}$ water masers are not a clear confirmation of high-mass star formation as they are also found towards sites where intermediate mass $\left(4-8 M_{\odot}\right)$ stars are forming. Water masers are thought to originate in the shocked gas associated with outflows and are collisionally pumped (e.g., Kylafis \& Pavlakis 1992). Core masses derived from $\mathrm{NH}_{3}$ range from $5 M_{\odot}$ to $\sim 516 M_{\odot}$, with a median value of $\sim 45 M_{\odot}$. Seven cores have masses greater than $100 M_{\odot}$ and for a typical star formation efficiency of 0.3 (c.f. Lada \& Lada 2003) are likely to form stars with masses greater than $8 M_{\odot}$.

We have confirmed that protostellar objects are likely forming in the arms of the filament and that the $\mathrm{H}_{\mathrm{II}}$ region is clearly influencing the conditions in the bulk of the cloud, as evidenced by the temperature gradients and gas-morphology. Whether the formation of the new stars is triggered by the expansion of the $\mathrm{H}$ il region is difficult to prove categorically, but to be consistent requires evidence of an "age gradient", as argued by Persi et al. (1994). Recent 450- $\mu \mathrm{m}$ continuum observations (André et al. 2008) partially resolved the $1.2-\mathrm{mm}$ continuum SIMBA clumps and detected continuum emission counterparts to the $\mathrm{NH}_{3}$ cores. Based on a "protostellar mass envelope vs. bolometric luminosity diagram" (hereafter $M_{\mathrm{env}}-L_{\mathrm{bol}}$ diagram), André et al. (2008) estimated the evolutionary stage of these candidate massive protostars and positioned them on evolutionary tracks for high mass star formation. Interestingly, S3-a and S3-b, the closest candidate protostars to the $\mathrm{H}$ iा region, appear to be the most evolved objects on the $M_{\text {env }}-L_{\text {bol }}$ diagram. In contrast, the other candidate protostars, those in the arms, appear to be less evolved on the diagram and are possibly class 0 -type protostars. The non detection of ionised gas toward these protostars confirms their very early evolutionary stage. All detected protostars will evolve into stars of masses between 15 and $50 M_{\odot}$ according to the evolutionary tracks on the $M_{\mathrm{env}}-L_{\mathrm{bol}}$ diagram.

Another signature of early phases of star formation is the evidence for collapsing cores. On examination of the relative molecular abundances, SIMBA clump S4 stands out as having an over-abundance of $\mathrm{N}_{2} \mathrm{H}^{+}$and an under-abundance of $\mathrm{CO}$. Such a chemical signature is typical of dense and cold regions, which is often found towards collapsing cores. We searched for signatures of inflowing motions in the $\mathrm{NH}_{3}$ line profiles. Asymmetries on the order of $\sim 10$ per cent are observed between the inner satellites for discrete regions in clump S4.

High-mass star formation is clearly underway in the dusty arms of the NGC 3576 filament. Evidence for interaction through temperature gradients as well as an evolutionary sequence for the embedded protostars from the $\mathrm{H}$ II region to the arms, is consistent with the hypothesis that the $\mathrm{H}$ II region is 


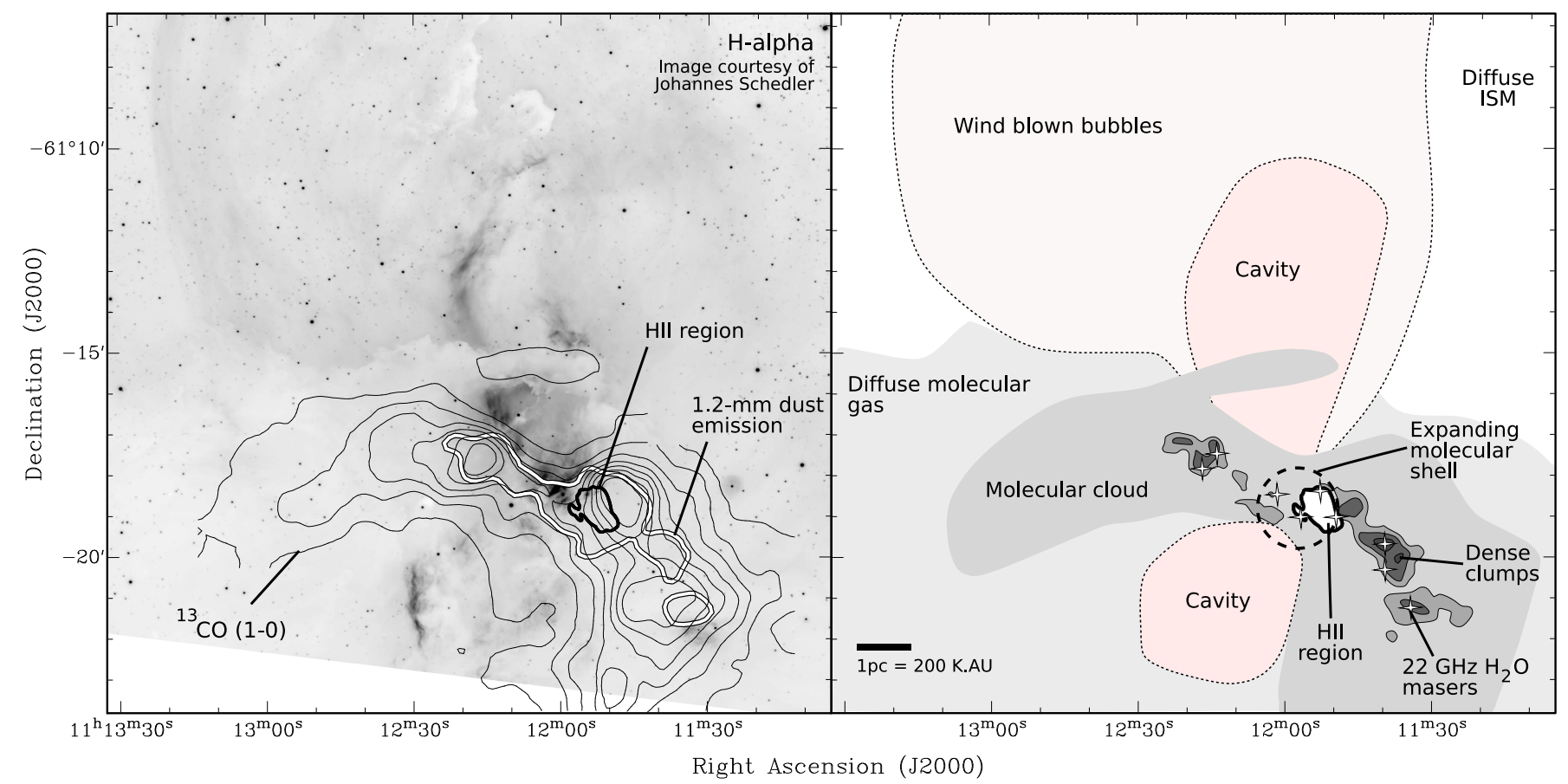

Fig. 16. Schematic of the environment of NGC 3576 (right panel). ${ }^{13} \mathrm{CO}$ integrated intensity contours tracing the extended molecular cloud, overlaid on a $\mathrm{H}-\alpha$ image (left panel), which highlights the large scale structure of the interstellar medium around the star-forming complex. The molecular emission is correlated exactly with the extincted regions of the H- $\alpha$. Loops of H- $\alpha$ emission are seen to extend north and south from the vicinity of the ionised $\mathrm{H}$ II region (thick black contour), likely shaped by the energetic young cluster at the heart of the complex. The ionised gas is expanding into the molecular cloud, sweeping up a clumpy shell of gas and heating the filamentary cloud in which it is embedded. Dense cores containing tens to hundreds of solar masses have formed along the length of the cloud and exhibit $22 \mathrm{GHz}$ water maser emission, signifying star-formation in progress.

triggering star formation in NGC 3576. The geometry of the $\mathrm{H}$ in region and associated clumps does not resemble the typical collect and collapse scenario, hence the observations are also in agreement with spontaneous star formation, at least in the clumps further away from the ionisation front. However, it is also possible that the $\mathrm{H}$ II region is responsible for initiating star formation in these clumps via shock driven globule squeezing. Further investigation is necessary to distinguish between these two cases.

\section{Summary and conclusions}

We have mapped the molecular environment of the giant $\mathrm{H}$ II region $\mathrm{NGC} 3576$ in lines of $\mathrm{NH}_{3}, \mathrm{CO}, \mathrm{HCO}^{+}, \mathrm{CS}$ and $\mathrm{N}_{2} \mathrm{H}^{+}$. In addition, we have searched for $\mathrm{UCH}$ iI regions via $23 \mathrm{GHz}$ free-free emission and for $22 \mathrm{GHz}$ water masers. Figure 16 summarises our observational results as a schematic, overlaid on an amateur H- $\alpha$ image (Schedler, 2009) which illustrates the large scale structure of the ISM in the region. Our main conclusions are as follows:

1. We have detected molecular emission from all clumps identified in the 1.2-mm SIMBA map (Hill et al. 2005). Dense gas tracers $\left(\mathrm{NH}_{3}, \mathrm{~N}_{2} \mathrm{H}^{+}\right.$and $\left.\mathrm{CS}\right)$ follow the morphology of the thermal dust emission, except within the $\mathrm{H}$ II region, where the $1.2-\mathrm{mm}$ emission is highly contaminated by free-free continuum emission. Molecules with low and intermediate critical densities or with high abundances $\left(\mathrm{CO}, \mathrm{HCO}^{+}\right)$trace the extended envelope well.

2. We searched for UCH II regions in the arms of the filament via their $23 \mathrm{GHz}$ emission, but found none down to a detection limit of $0.5 \mathrm{mJy} /$ beam. The central $\mathrm{H}$ II region was mapped and exhibited a similar morphology to the earlier 3.4-cm image by de Pree et al. (1999).

3. Six new sites of $22 \mathrm{GHz}$ water maser emission were detected, all of which lie adjacent to discrete $\mathrm{NH}_{3}$ cores in the arms of the filament. Most of the new masers have flux densities less than ten Janskys, compared to the three known sites in the $\mathrm{H}$ II region, which have flux densities between 40-640 Jy. Of all the new masers, the site in clump S5 has the greatest range of velocities and warrants further investigation.

4. $\mathrm{NH}_{3}(1,1)$ and $(2,2)$ were used to derive the kinetic temperature of the dense gas. We find a temperature gradient exists in the western arm, with temperatures ranging from $\sim 11 \mathrm{~K}$ at the extremes to $\geq 30 \mathrm{~K}$ adjacent to the $\mathrm{H}$ in region. Clearly, the $\mathrm{H}$ II region is responsible for the heating.

5. There is clear evidence in ${ }^{13} \mathrm{CO}$ and $\mathrm{C}^{18} \mathrm{O}$ for an expanding swept-up shell of diameter $\sim 1^{\prime}$ completely encompassing the $\mathrm{H}$ iI region.

6. The $\sim 11^{\prime \prime}$ resolution $\mathrm{NH}_{3}(1,1)$ emission was decomposed into twenty-five individual cores, ranging in mass from $5 M_{\odot}$ to $516 M_{\odot}$, when scaled to the 2-D clump masses derived from the $450 \mu \mathrm{m}$ P-ArTéMiS map.

7. $M_{\text {corrected }} / M_{\text {vir }} \geq 1$ for all $\mathrm{NH}_{3}$ cores, implying they are at least gravitationally bound. Seveneen cores have $M_{\text {corrected }} / M_{\text {vir }} \geq 3$ and to avoid collapsing under self-gravity require support from internal magnetic fields with strengths between $\sim 1 \mathrm{mG}$ and $\sim 40 \mathrm{mG}$. These values are on average higher than measured in recent observations of massive starformation regions and suggest that some of the cores may be collapsing.

8. Clump S4 in Fig. 1 is over-abundant in $\mathrm{N}_{2} \mathrm{H}^{+}$and shows evidence for depletion of $\mathrm{CO}$, consistent with the expected chemistry in a cold, dense clump. We examined 
our $\mathrm{NH}_{3}$ data for line-profile asymmetries indicating bulk gas motions and found distinct regions with blue profiles, tentatively indicating inflowing gas.

9. The filament displays a complex velocity structure, with two main components in each arm seen in the ${ }^{13} \mathrm{CO}$ data. Molecules tracing high density gas show that a single component at $V_{\mathrm{LSR}}=-24 \mathrm{~km} \mathrm{~s}^{-1}$ dominates the cloud.

10. The positions of stars with infrared-excess, indicative of circumstellar disks, are anti-correlated with the dense molecular gas, implying star formation has not yet advanced far enough to be visible in the near-infrared. Alternatively, there is likely sufficient extinction to hide such stars embedded within the molecular cloud.

From the above evidence it is clear that star formation is underway across the whole of the NGC 3576 filament and that the $\mathrm{H}$ II region is influencing the physical conditions in the bulk of the molecular gas. Very young massive protostellar objects, some equivalent to low-mass class-0 protostars, are forming within the arms of the filament. Masses and luminosities of cores with 1.2-mm, $450 \mu \mathrm{m}$ and infrared data have been characterised by André et al. (2008). Objects adjacent to the $\mathrm{H}$ II region appear to be more evolved, implying an evolutionary gradient. The $\mathrm{H}$ II region is expanding into the cloud, dissipating the gas on its eastern edge and is possibly the trigger for star-formation in the nearest clumps. However, the observational data is also consistent with spontaneous star formation, at least in the outlying regions.

Acknowledgements. The Mopra radio telescope is part of the Australia Telescope which is funded by the Commonwealth of Australia for operations as a National Facility managed by CSIRO. During 2002-2005 the Mopra telescope was operated through a collaborative arrangement between the University of New South Wales and the CSIRO.

We wish to thank the Australian research council and UNSW for grant support. C.R.P. was supported by a School of Physics Scholarship during the course of his $\mathrm{PhD}$.

V. Minier and F. Herpin acknowledge the use of a French-Australian Science $\&$ Technology (FAST) Program grant. The FAST program is jointly managed by the Department of Innovation, Industry, Science \& Research and its French counterparts, the Ministry of Higher Education and Research and the Ministry of Foreign and European Affairs.

P.A.J. acknowledges partial support from Centro de Astrofísica FONDAP 15010003 and the GEMINI-CONICYT FUND.

Many thanks also to Johannes Schedler, who kindly provided his H- $\alpha$ image of NGC 3576.

We are also grateful to the anonymous referee for very thorough comments and discussions that helped improve the presentation.

\section{Appendix A: Physical properties from molecular lines}

This work makes heavy use of standard methods to derive physical properties from molecular line data. Here we summarise the methodology used and gather the equations and constants in one place. All calculations were performed in SI units.

\section{A.1. Kinetic temperature from $\mathrm{NH}_{3}$}

Firstly, we calculate the optical depth of the $\mathrm{NH}_{3}(1,1)$ transition via a comparison of brightness temperature $T_{\mathrm{B}}$ in the main and satellite lines:

$\frac{T_{\mathrm{B}, \mathrm{m}}}{T_{\mathrm{B}, \mathrm{s}}}=\frac{1-\mathrm{e}^{-\tau_{\mathrm{m}}}}{1-\mathrm{e}^{-a \tau_{\mathrm{m}}}}$.

The subscripts " $\mathrm{m}$ " and "s" indicate the main and satellite groups, respectively, and " $a$ " is the theoretical $T_{\mathrm{B}, \mathrm{s}} / T_{\mathrm{B}, \mathrm{m}}$ ratio under optically thin conditions. For $\mathrm{NH}_{3}(1,1)$, the fractional intensity in the main group is 0.502 and in individual inner and outer satellite lines is 0.139 and 0.111 , respectively. In practise we used the hyperfine fitting utility of the CLASS software package to simultaneously fit the five groups. It is often difficult to measure the optical depth directly from the $\mathrm{NH}_{3}(2,2)$ line profile as the signal-to-noise on the satellites is usually poor. Instead, $\tau_{2,2}$ may be calculated from the brightness temperatures of the $(2,2)$ and $(1,1)$ main groups:

$\tau_{2,2}=-\frac{1}{f_{2,2}} \ln \left[1-\frac{T_{B_{2,2} \text { main }}}{T_{B_{1,1} \text { main }}}\left(1-\mathrm{e}^{-\tau_{1,1}^{\mathrm{tot}} f_{1,1}}\right)\right]$,

where $f_{1,1}=0.502$ and $f_{2,2}=0.796$ are the fractional intensities in the $(1,1)$ and $(2,2)$ main groups, respectively, compared to their satellites. The rotational temperature is found from the optical depth ratio $\tau_{2,2} / \tau_{1,1}$ via:

$T_{\text {rot }}=-41 \mathrm{~K} / \ln \left(\frac{9}{20} \frac{\tau_{2,2}}{\tau_{1,1}} \frac{\Delta v_{2,2}}{\Delta v_{1,1}}\right)$,

where $\tau_{J, K}$ and $\Delta v_{J, K}$ are the total optical depth and the full-width half-maximum $(F W H M)$ linewidth of the $\mathrm{NH}_{3}(J, K)$ transition. Finally, the rotational temperature may be converted directly to a kinetic temperature $T_{\text {kin }}$ via:

$T_{\text {rot }}=\frac{T_{\text {kin }}}{\left[1+\left(T_{\text {kin }} / 41 K\right) \ln \left(1+C_{23} / C_{21}\right)\right]}$.

Danby et al. (1988) have used large velocity gradient (LVG) models to calculate the collisional coefficients $C_{23}$ and $C_{21}$, for kinetic temperatures ranging from 5-300 K. To derive Eq. (A.4) we assume that only the $J, K=(1,1),(2,1)$ and $(2,2)$ transitions are involved. At low temperatures this assumption is valid, however, above $T_{\text {rot }} \sim 30 \mathrm{~K}$ the excitation of $\mathrm{NH}_{3}$ to higher energy levels becomes non-negligible. This means that the above $T_{\text {rot }} \sim 30 \mathrm{~K}$ the kinetic temperature found via comparison of the $\mathrm{NH}_{3}(1,1)$ and $(2,2)$ lines becomes unreliable. Tafalla et al. (2004) have investigated the accuracy of this method and find that kinetic temperatures derived are accurate to better than 5 percent below $20 \mathrm{~K}$.

\section{A.2. Column density and core mass}

If the optical depth and excitation temperature in any $(J, K)$ transition are known, the total column density of emitting molecules may be calculated assuming local thermal equilibrium (LTE) via the equation

$N_{J, K}=\frac{8 k \pi v_{J, K}^{2}}{A_{\mathrm{ul}} h c^{3}} \int_{-\infty}^{\infty} T_{b} \mathrm{~d} v\left(\frac{\tau}{1-\mathrm{e}^{-\tau}}\right) \frac{\mathrm{e}^{\left(E_{J, K}\right) / k T}}{g_{J, K} Q\left(T_{\mathrm{rot}}\right)}$,

where $Q\left(T_{\text {rot }}\right)$ is the partition function. The exact partition function is the sum over all the levels however, this can be approximated by an exponential fit to the discrete values in the Pickett, et al. (1998) spectral line catalogue. For $\mathrm{NH}_{3}$ this is:

$Q(T)=0.1266 T^{1.48}$.

\section{A.3. $\mathrm{CO}$ and $\mathrm{HCO}^{+}$}

Physical conditions were calculated on a pixel-by-pixel basis in the Mopra data. Using the regrid task in MIRIAD, the CO datacubes were first resampled to the same pixel-scale in RA, Dec 
Table A.1. Molecular constants ${ }^{\alpha}$.

\begin{tabular}{lccccc}
\hline \hline Species & $\begin{array}{c}\text { Transition } \\
\left(J_{K}\right)\end{array}$ & $\begin{array}{c}\text { Frequency } \\
(\mathrm{GHz})\end{array}$ & $\begin{array}{c}E_{\mathrm{u}} / \mathrm{k} \\
(K)\end{array}$ & $\begin{array}{c}A_{\mathrm{ul}} \\
\left(\mathrm{s}^{-1}\right)\end{array}$ & $\begin{array}{c}n_{\text {crit }} \beta \\
\left(\mathrm{cm}^{-3}\right)\end{array}$ \\
\hline $\mathrm{HCO}^{+}$ & $1 \rightarrow 0$ & 89.188526 & 4.280 & $3.020 \times 10^{-5}$ & $3.0 \times 10^{5}$ \\
$\mathrm{H}^{13} \mathrm{CO}^{+}$ & $1 \rightarrow 0$ & 86.754330 & 4.160 & $2.800 \times 10^{-5}$ & $3.0 \times 10^{5 \dagger}$ \\
${ }^{13} \mathrm{CO}$ & $1 \rightarrow 0$ & 110.210353 & 5.288 & $6.389 \times 10^{-8}$ & $6.5 \times 10^{2 \dagger}$ \\
$\mathrm{C}^{18} \mathrm{O}$ & $1 \rightarrow 0$ & 109.782173 & 5.267 & $4.961 \times 10^{-7}$ & - \\
$\mathrm{CS}$ & $2 \rightarrow 1$ & 97.980950 & 7.052 & $1.703 \times 10^{-5}$ & $2.0 \times 10^{5}$ \\
$\mathrm{~N}_{2} \mathrm{H}^{+}$ & $1_{1} \rightarrow 0_{1}$ & 93.171880 & 4.471 & $3.654 \times 10^{-5}$ & $2 \times 10^{5}$ un \\
& $1_{2} \rightarrow 0_{1}$ & 93.173700 & - & - & - \\
& $1_{0} \rightarrow 0_{1}$ & 93.176130 & - & - & - \\
$\mathrm{NH}_{3}$ & $1_{1} \rightarrow 1_{1^{\prime}}^{\prime}$ & 23.694480 & 17.215 & $1.67 \times 10^{-7}$ & $2 \times 10^{4} \mathrm{sw}$ \\
& $2_{2} \rightarrow 2_{2^{\prime}}$ & 23.722630 & 63.866 & $2.29 \times 10^{-7}$ & \\
\hline
\end{tabular}

${ }^{\alpha}$ Unless otherwise noted, all values quoted here are sourced from the NASA Jet Propulsion Lab (JPL) spectral line database (Pickett, et al. 1998); ${ }^{\beta}$ Critical density values marked with a $\dagger$ are calculated from the formula $n_{\text {crit }}=A_{\mathrm{ul}} / \gamma_{\mathrm{H}_{2}}$, where $\gamma_{\mathrm{H}_{2}}$ is the "standard" collisional rate coefficient for $\mathrm{H}_{2}$, assumed equal to $10^{10} \mathrm{~cm}^{3} \cdot \mathrm{s}^{-1} \cdot \mathrm{H}_{2}-$ molecules $^{-1}$. Other values are referenced as follows: ${ }^{u n}$ Ungerechts et al. (1997), ${ }^{\text {sw }} \mathrm{Swade}$ (1989).

and velocity. The maths task was used to perform all subsequent calculations. For each spatial pixel we calculated the optical depth as a function of frequency using a modified version of the isotope ratio (Bourke et al. 1997):

$\frac{T_{\mathrm{MB}}\left(\mathrm{C}^{18} \mathrm{O}\right)}{T_{\mathrm{MB}}\left({ }^{13} \mathrm{CO}\right)}=\frac{1-\mathrm{e}^{-\left(\tau_{18} / X\right)}}{1-\mathrm{e}^{-\tau}{ }_{13}}$,

In the above equation, $T_{\mathrm{MB}}$ is the peak main beam brightness temperature and $\mathrm{X}$ is the abundance ratio of $\left[{ }^{13} \mathrm{CO}\right] /\left[\mathrm{C}^{18} \mathrm{O}\right]$ assumed to be constant. During the calculation pixels with insufficient emission were blanked, resulting in a map of ${ }^{13} \mathrm{CO}$ optical depth with real values wherever $\mathrm{C}^{18} \mathrm{O}$ emission was detected. The expected $\left[{ }^{13} \mathrm{CO} / \mathrm{C}^{18} \mathrm{O}\right]$ ratio in giant molecular clouds is $\sim 11.5$ (see Goldsmith et al. 1997 and references therein), however, we found that this value resulted in negative optical depths in some parts of the map. The lowest abundance ratio that did not result in negative optical depths was $\sim 16$. This value was used in our calculations and should be considered a lower limit. Optical depths ranged from $0.1 \leq \tau \leq 4.4$. Using the ${ }^{13} \mathrm{CO}$ optical depth and peak brightness temperature maps as input, we then constructed an excitation temperature map via:

$T_{\mathrm{ex}}=\frac{h v_{u}}{k}\left[\ln \left(1+\frac{\left(h v_{u} / k\right)}{T_{13} / C_{\tau}+J_{v}\left(T_{b g}\right)}\right)\right]^{-1}$,

where $C_{\tau}=\left(1-\mathrm{e}^{-\tau_{13}}\right)$. At this stage we blanked pixels with spuriously excitation temperatures $\left(T_{\mathrm{ex}}<4 \mathrm{~K}\right.$ and $\left.T_{\mathrm{ex}}>40 \mathrm{~K}\right)$ due to noisy $\mathrm{C}^{18} \mathrm{O}$ data which had passed our initial signal-tonoise cutoff. Assuming a thermalised population with $T_{\text {kin }}=T_{\text {ex }}$, a column density map was be produced via Eq. (A.5). The partition function $\mathrm{Q}(\mathrm{T})$ for ${ }^{13} \mathrm{CO}$ has been measured exactly at discrete values by Pickett, et al. (1998) spectral line catalogue. Intermediate values were determined via an exponential fit, which for $\mathrm{CO}$ is:

$Q\left(T_{\text {kin }}\right)=0.3968 T_{\text {kin }}$.

The CO column density map is presented in Fig. 10a.

We calculated the column-density of $\mathrm{HCO}^{+}$using the same procedure, and a partition function of the form:

$Q\left(T_{\text {kin }}\right)=0.4798 T_{\text {kin }}$.

The $\left[\mathrm{HCO}^{+}\right] /\left[\mathrm{H}^{13} \mathrm{CO}^{+}\right]$was set to 40 for these calculations.

\section{A.4. $\mathrm{N}_{2} \mathrm{H}^{+}$and CS}

A slightly different approach was taken for the $\mathrm{N}_{2} \mathrm{H}^{+}$data. In a similar manner to $\mathrm{NH}_{3}$, the hyperfine structure in the spectrum of $\mathrm{N}_{2} \mathrm{H}^{+}(1-0)$ allows the direct determination of optical depth. We fit all spectra in the $\mathrm{N}_{2} \mathrm{H}^{+}$data-cube using the HFS method in CLASS and found that the $\mathrm{N}_{2} \mathrm{H}^{+}$emission was consistent with it being optically thin $(\tau \ll 0.1)$ over the entire cloud. Hence, no independent estimate could be made of the excitation temperature. $\mathrm{N}_{2} \mathrm{H}^{+}$and $\mathrm{NH}_{3}$ have similar critical densities and, being nitrogen bearing molecules, are often detected under similar conditions. We calculated the column density of $\mathrm{N}_{2} \mathrm{H}^{+}$assuming excitation temperatures equal to the $\mathrm{NH}_{3}$ rotational temperatures. In practise, we smoothed the $T_{\text {rot }}\left(\mathrm{NH}_{3}\right)$ map to the same resolution as the Mopra $\mathrm{N}_{2} \mathrm{H}^{+}$data. We then produced a column density map by combining this $T_{\text {ex }}$ map with the $\mathrm{N}_{2} \mathrm{H}^{+}$integrated map via Eq. (A.5) in the limit that $\tau \ll 0$. The partition function for $\mathrm{N}_{2} \mathrm{H}^{+}$was again interpolated from the values in the Pickett, et al. (1998) catalogue:

$Q\left(T_{\text {kin }}\right)=4.198 T_{\text {kin }}$

A column density map of CS was prepared in a similar manner, although we note that our assumption of optically thin emission may not be valid. Judging by the non-Gaussian shapes of some line profiles, regions with strong emission may have significant optical depths.

\section{References}

Aikawa, Y., Ohashi, N., Inutsuka, S.-I., Herbst, E., \& Takakuwa, S. 2001, ApJ, 552,639

Allen, C. W. 1973, Astrophysical quantities (London: University of London, Athlone Press, |c1973, 3rd ed.)

Allen, L. E., Burton, M. G., Ryder, S. D., Ashley, M. C. B., \& Storey, J. W. V. 1999, MNRAS, 304, 98

André, P., Minier, V., Gallais, P., et al. 2008, A\&A, 490, L27

Barbosa, C. L., Damineli, A., Blum, R. D., et al. 2003, AJ, 126, 2411

Bergin, E. A., Alves, J., Huard, T., et al. 2002, ApJ, 570, L101

Beuther, H., Walsh, A., Schilke, P., et al. 2002, A\&A, 390, 289

Bot, C., Boulanger, F., Rubio, M., et al. 2007, A\&A, 471, 103

Bourke, T. L., Hyland, A. R., Robinson, G., James, S. D., \& Wright, C. M. 1995, MNRAS, 276, 1067

Bourke, T. L., Garay, G., Lehtinen, K. K., et al. 1997, ApJ, 476, 781

Caselli, P., Myers, P. C., \& Thaddeus, P. 1995, ApJ, 455, L77

Caswell, J. L. 2004, MNRAS, 351, 279

Caswell, J. L., Batchelor, R. A., Forster, J. R., et al. 1989, Austr. J. Phys., 42, 331 
Caswell, J. L., \& Phillips, C. J. 2008, MNRAS, 386, 1521

Cesaroni, R., Walmsley, C. M., \& Churchwell, E. 1992, A\&A, 256, 618

Cheung, L. H., Frogel, J. A., Hauser, M. G., et al. 1980, ApJ, 240, 74

Curran, R. L., \& Chrysostomou, A. 2007, MNRAS, 382, 699

Danby, G., Flower, D. R., Valiron, P., Schilke, P., \& Walmsley, C. M. 1988, MNRAS, 235, 229

de Pree, C. G., Nysewander, M. C., \& Goss, W. M. 1999, AJ, 117, 2902

Deharveng, L., Lefloch, B., Kurtz, S., et al. 2008, A\&A, 482, 585

Deharveng, L., Zavagno, A., Schuller, F., et al. 2009, A\&A, 496, 177

Elmegreen, B. G. 1998, in ASP Conf. Ser. 148: Origins, ed. C. E. Woodward, J. M. Shull, \& H. A. Thronson, Jr., 150

Evans, II, N. J. 1999, ARA\&A, 37, 311

Frogel, J. A., \& Persson, S. E. 1974, ApJ, 192, 351

Genzel, R. 1991, in NATO ASIC Proc. 342: The Physics of Star Formation and Early Stellar Evolution, ed. C. J. Lada, \& N. D. Kylafis, 155

Goldsmith, P. F., Bergin, E. A., \& Lis, D. C. 1997, ApJ, 491, 615

Greenhill, L. J., Booth, R. S., Ellingsen, S. P., et al. 2003, ApJ, 590, 162

Han, J. L., Manchester, R. N., Lyne, A. G., Qiao, G. J., \& van Straten, W. 2006, ApJ, 642, 868

Hanson, M. M., \& Conti, P. S. 1993, Bull. Amer. Astron. Soc., 25, 1456

Henning, T., Begemann, B., Mutschke, H., et al. 1995, A\&AS, 112, 143

Hill, T., Burton, M. G., Thompson, M. A., et al. 2005, MNRAS, 363, 405

Hjellming, R. M. 1966, ApJ, 143, 420

Ho, P. T. P., \& Townes, C. H. 1983, ARA\&A, 21, 239

Ho, P. T. P., Martin, R. N., Myers, P. C., \& Barrett, A. H. 1977, ApJ, 215, L29

Hollenbach, D. J., \& Tielens, A. G. G. M. 1999, Rev. Mod. Phys., 71, 173

Karr, J. L., \& Martin, P. G. 2003, ApJ, 595, 900

Kutner, M. L., \& Ulich, B. L. 1981, ApJ, 250, 341

Kylafis, N. D., \& Pavlakis, K. G. 1992, ApJ, 400, 344

Lada, C. J., \& Lada, E. A. 2003, ARA\&A, 41, 57

Ladd, E. F., Purcell, C. R., Wong, T., et al. 2005, PASA, 22, 62

Langer, W. D., \& Penzias, A. A. 1990, ApJ, 357, 477

MacLaren, I., Richardson, K. M., \& Wolfendale, A. W. 1988, ApJ, 333, 821

Maercker, M., Burton, M. G., \& Wright, C. M. 2006, A\&A, 450, 253

Moneti, A. 1992, A\&A, 259, 627

Moorwood, A. F. M., \& Salinari, P. 1981, A\&A, 102, 197

Motte, F., Andre, P., \& Neri, R. 1998, A\&A, 336, 150
Norris, R. P., Whiteoak, J. B., Caswell, J. L., Wieringa, M. H., \& Gough, R. G. 1993, ApJ, 412, 222

Oliva, E., \& Moorwood, A. F. M. 1986, A\&A, 164, 104

Park, Y.-S. 2001, A\&A, 376, 348

Peretto, N., André, P., \& Belloche, A. 2006, A\&A, 445, 979

Persi, P., Roth, M., Tapia, M., Ferrari-Toniolo, M., \& Marenzi, A. R. 1994, A\&A, 282,474

Pickett, H. M., Poynter, R. L., Cohen, E. A., et al. 1998, J. Quant. Spec. Radiat. Transf., 60, 883

Pillai, T., Wyrowski, F., Carey, S. J., et al. 2006, A\&A, 450, 569

Pirogov, L., Zinchenko, I., Caselli, P., Johansson, L. E. B., \& Myers, P. C. 2003, A\&A, 405, 639

Pirogov, L., Zinchenko, I., Caselli, P., et al. 2007, A\&A, 461, 523

Rathborne, J. M., Jackson, J. M., \& Simon, R. 2006, ApJ, 641, 389

Rawlings, J. M. C., Redman, M. P., Keto, E., et al. 2004, MNRAS, 351, 1054

Robitaille, T. P., Whitney, B. A., Indebetouw, R., Wood, K., \& Denzmore, P. 2006, ApJS, 167, 256

Robitaille, T. P., Whitney, B. A., Indebetouw, R., et al. 2007, ApJS, 169, 328

Rydbeck, O. E. H., Sume, A., Hjalmarson, A., et al. 1977, ApJ, 215, L35

Sault, R. J., Teuben, P. J., \& Wright, M. C. H. 1995, in Astronomical Data Analysis Software and Systems IV, ed. R. A. Shaw, H. E. Payne, \& J. J. E. Hayes, ASP Conf. Ser. 77, 433

Stutzki, J., \& Winnewisser, G. 1985, A\&A, 144, 13

Swade, D. A. 1989, ApJ, 345, 828

Tafalla, M., Myers, P. C., Caselli, P., et al. 2004, A\&A, 416, 191

Townes, C. H., Genzel, R., Watson, D. M., et al. 1983, ApJ, 269, L11

Townsley, L. K. 2006, ArXiv Astrophysics e-prints

Ungerechts, H., Walmsley, C. M., \& Winnewisser, G. 1980, A\&A, 88, 259

Ungerechts, H., Winnewisser, G., \& Walmsley, C. M. 1986, A\&A, 157, 207

Ungerechts, H., Bergin, E. A., Goldsmith, P. F., et al. 1997, ApJ, 482, 245

Urquhart, J. S., Thompson, M. A., Morgan, L. K., et al. 2006, A\&A, 450, 625

Wang, Y., Zhang, Q., Pillai, T., Wyrowski, F., \& Wu, Y. 2008, ApJ, 672, L33

Ward-Thompson, D., Motte, F., \& Andre, P. 1999, MNRAS, 305, 143

White, G. J., Nelson, R. P., Holland, W. S., 1999, A\&A, 342, 233

Whitney, B. A., Indebetouw, R., Babler, B. L., et al. 2004, ApJS, 154, 315

Zavagno, A., Deharveng, L., Comerón, F., et al. 2006, A\&A, 446, 171

Zhu, L.-B., Yang, J., \& Wang, M. 2007, Chin. Astron. Astrophys., 31, 387 